\title{
Radio properties of FIR-megamaser nuclei ${ }^{\star}$
}

\author{
W. A. Baan ${ }^{1}$ and H.-R. Klöckner ${ }^{1,2}$ \\ 1 ASTRON, Westerbork Observatory, PO Box 2, 7990 AA Dwingeloo, The Netherlands \\ e-mail: baan@astron.nl \\ 2 University of Oxford, Denys Wilkinson Building, Keble Road, Oxford OX1 3RH, UK \\ e-mail: hrk@astro.ox.ac.uk
}

Received 8 November 2004 / Accepted 7 December 2005

\section{ABSTRACT}

\begin{abstract}
Aims. Radio data on the nuclear emissions have been used to characterize the dominant nuclear activity in a sample of FIR (ultra-) luminous galaxies and the subgroup of known $\mathrm{OH}$ Megamasers. This study complements an earlier study of the optical classification of these Megamaser nuclei.

Methods. Classification of the radio activity in the nuclei is based on three critical parameters: the radio brightness temperature, the radio spectral index, and the ratio of FIR and radio fluxes. A first method gives equal weight to the three parameters and a second method uses a weighted function to classify the nuclei.

Results. The present sample shows that only $43 \%$ of the sample shows some - weak or strong - AGN characteristics. About $66 \%$ of the $\mathrm{OH}-\mathrm{MM}$ sample and $81 \%$ of the non-OH-MM sample can be actually classified as Starburst-dominated sources. Radio diagnostic diagrams using these diagnostic parameters show a continuous distribution ranging between AGN-dominated and SBN-dominated sources. The diagnostic diagrams also support the notion that AGNs and starbursts coexist in the nuclei.

Conclusions. A comparison of the radio and optical classifications shows a consistency in the extreme cases of clear SBN and AGNs. A significant part of the sources with optical AGN-like activity have an SBN classification in the radio. The discrepant classifications are discussed in order to arrive at a final classification of the dominant power source in the nucleus.
\end{abstract}

Key words. galaxies: nuclei - galaxies: Seyfert - galaxies: starburst - radio continuum: galaxies

\section{Introduction}

The radio characteristics of extragalactic sources from the IRAS Point Source Catalog have been interpreted to be due to either a Starburst Nucleus (SBN) with circum-nuclear/compact star-formation or the presence of an Active Galactic Nucleus (AGN). The primary distinction between these sources lies in the nature of the underlying energy source for the optical, radio and infrared emissions. Although most unresolved $\left(\leq 1^{\prime \prime}\right)$ nearby extragalactic radio sources with optical luminosities of normal galaxies are generally considered as AGNs, a physical source size of $\leq 1 \mathrm{pc}$ would be a stricter operational definition (Condon 1992). A total of $63 \%$ of the ultra-luminous FIR galaxies from the IRAS Bright Galaxy Sample (IRAS BGS) with $L_{\mathrm{FIR}} \geq 10^{11.25} L_{\odot}$ and $S_{60 \mu \mathrm{m}}>5.24$ Jy show diffuse radio emission resulting from starburst activity (Condon et al. 1991a, "CHYT"). CHYT argue that another 35\% of the sample sources are compact and ultra-luminous nuclear starbursts

* Appendices A and B are only available in electronic form at http://www . edpsciences.org (with or without an AGN present), that are optically thick due to free-free absorption.

The radio and FIR luminosities of starburst galaxies are strongly correlated since the same population of massive stars produces thermal FIR emission from HII regions and subsequently non-thermal emission from supernovae. This nearlinear relation holds for 5 orders of magnitude (Price \& Duric 1992; Yun et al. 2001). This correlation is directly related to the connection between star formation and cosmic-ray production, although the radio and the FIR are produced in different stages of the starburst cycle. The FIR-Radio ratio or the $q$-ratio is defined as the logarithm of the ratio of the FIR flux density and the radio flux density at e.g. $4.85 \mathrm{GHz}$, and is indicative of the nature and efficiency of the underlying heating process (Helou et al. 1985). Starburst galaxies show consistently high $q$-ratios of about 2.75. When a large fraction of the radio emission is powered by the AGN, while leaving the FIR unaffected, the $q$-ratio becomes lower. This increased "radio-loud-ness" makes sources deviate from the simple relation between radio and FIR luminosities. The $q$-ratio may thus be used to select "monster-AGN" sources (Yun et al. 2001). 
Besides the value of the $q$-ratio, a number of other spectral and structural characteristics may help to distinguish between the two extremes in the nature of radio sources. First, the spectral shape of the radio spectrum distinguishes between starburst and AGNs. These composite thermal/non-thermal spectra of compact starburst-dominated sources may further be affected by free-free absorption. The $L / C$ band spectral indices of most galaxies fall within a narrow range centered on $\alpha=$ 0.75 (Condon 1983). AGN-dominated sources have intrinsically flat $(\alpha \leq 0.5)$ radio spectra (Condon \& Broderick 1988). Second, AGNs are likely to be very compact and have very high brightness temperatures. Starbursts show a centrally brightened but diffuse structure due to the distribution of massive stars broadened by cosmic ray transport. About $75 \%$ of the radio galaxy population can thus be classified on the basis of their radio morphology in low-resolution maps, because many sources have indeed significant starburst contributions (Condon $\&$ Broderick 1988). Classifying the remaining sources requires higher resolution maps.

In this paper we consider the radio characteristics of primarily $\mathrm{OH}$ Megamaser galaxies at $1^{\prime \prime}$ and $0.3^{\prime \prime}$ resolution based on data taken with the Very Large Array (VLA) ${ }^{1}$ in the A-configuration. The Megamaser characteristics and those of the standard amplification model have been described elsewhere (Baan 1989, 1997; Henkel \& Wilson 1990; Henkel et al. 1992). The current sample of OH-MM and comparison sources covers a wide range of FIR luminosities and includes (ultra-) luminous infrared galaxies (LIRGs and ULIRGs). The sample is likely to cover a significant range of evolutionary history of the FIR sources (Baan 1988; Majewski et al. 1993).

\section{Observations and data reduction}

The data on the radio structures of the $\mathrm{OH}$ Megamasers have been obtained with the VLA A-array in November 1992. Twelve hours of observing time have been used to obtain $72 L$-band and $C$-band snapshots of 51 sources. Additional data from the literature provides further information on 22 sources. $L$-band and $C$-band snapshots lasted typically six minutes. The same phase calibrator was used for snapshots in two bands for each source. Two independent dual-polarization $L$-band observations having a $50 \mathrm{MHz}$ bandwidth were centered at 1385 and $1464 \mathrm{MHz}$. At $C$-band the observations were done at 4835 and $4885 \mathrm{MHz}$ also with a $50 \mathrm{MHz}$ bandwidth. In addition to the results of our observations, other data at 1.43 and $8.44 \mathrm{GHz}$ by Condon et al. (1990, 1991b, "CHYT") have been used to complement our data. As a result our total source sample consists of 51 sources with data at $1.4 \mathrm{GHz}$ and at 4.83 or $8.44 \mathrm{GHz}$. The OH absorber IR03134+0941 has only data at $1.43 \mathrm{GHz}$.

The data have initially been calibrated using the AIPS package. All imaging has been done using Uniform weighting and phase self-calibration. The beam size for Uniform weighted maps is typically $1.0^{\prime \prime} \times 1.0^{\prime \prime}$ for the $L$-band data and $0.3^{\prime \prime} \times$ $0.3^{\prime \prime}$ for the $C$-band data. The noise levels for the $L$-band

\footnotetext{
${ }^{1}$ The Very Large Array is a facility of the National Radio Astronomy Observatory and is operated under cooperative agreement with the National Science Foundation.
}

snapshots range from $0.2 \mathrm{mJy}$ beam $^{-1}$ to about $1 \%$ of the peak flux density; for the $C$-band snapshots they range from $0.10 \mathrm{mJy}$ beam $^{-1}$ to $1 \%$ of the peak flux density. The dynamic range in the maps varies from four to a few hundred.

The imaging results for only the (partially) resolved sources are presented in Fig. A.1. The contour levels are Level $\times$ $(\sqrt{2})^{n}(\mathrm{mJy} / \mathrm{beam})$, where $n=1,2,3$, etc. The lowest contour level is given within the frame of each graph. A tabular presentation for all available spectral and structural information is presented in Table B.1. The content of the columns has been explained in footnote 1 to the Table B.1. $H_{0}=75 \mathrm{~km} \mathrm{~s}^{-1} \mathrm{Mpc}^{-1}$ is assumed throughout this paper and our distance determination $D_{L}=\left(c z / H_{0}\right)(1+0.5 z)$ assumes a deceleration parameter of $q_{0}=0$. The $L$-band and $C$-band maps of sources that show some spatial extended structure and the ones deserving special mention are described in footnote 2 to Table B.1.

Table B. 2 provides the relevant information for the classification of the radio sources. The columns in the table are explained in the footnote.

The individual components of sources with clear substructure have been tabulated with separate entries for $T_{b}$ and for $\alpha$. For the $q$-values of these sources it was not possible to allocate reliably the low resolution (15") FIR $S_{60 \mu \mathrm{m}}+S_{100 \mu \mathrm{m}}$ flux components to the individual radio components. The $q$-ratios presented are based on the FIR flux and the sum of the 1.4 and $4.8 \mathrm{GHz}$ fluxes of the components; the $q$-values are the same for all radio components.

Twenty-two of our sample of 51 sources show extensions within the nuclear region, either in the form of a central disk or possible jet structures (see Fig. A.1). Seven sources IR05414+5840, IR13097-1531, IR16399+5900, IR17526+3253, and possibly IR22025+4205, as well as OH absorbers IR03134+0941 and IR16399-0937, show an extended (triple) structure embedded in a central disk structure. At higher $C$-band resolution, the nuclear emission in the sources IR13097-1531, IR16399-0937, and IR17526+3253 breaks up in smaller components, which may suggest that the emission results from (individual) SNRs that are the product of pockets of massive star formation with typical size of $145 \mathrm{pc}$. The majority of the sample sources have a partially resolved nuclear source, consistent with various combinations of nuclear starbursts and AGNs.

\section{How to classify radio galactic nuclei?}

In the following subsections, we consider the classifications of our sample sources by evaluating independent criteria for the radio brightness temperature at $1.4 \mathrm{GHz}$ and $4.85 \mathrm{GHz}$, the FIR-radio $q$-ratio at both frequencies, and the peak and integrated radio spectral index. These parameters have been used individually for radio classification schemes in the literature. In addition, an angular upper limit for the size of the nucleus has been used to classify AGNs. Because, the compact nuclear components in our sample are only partially resolved with sizes $<1^{\prime \prime}$ at 4.85 and $8.44 \mathrm{GHz}$, the corresponding spatial sizes could not really distinguish differences between a compact starburst and an AGN (Condon 1992). Based on the observed parameters, a nuclear classification code " $N C$ " has been devised 
using three criteria to reflect a galaxy's tendency towards SBN or AGN-dominance. If the galaxy meets all three criteria for AGN activity, $\mathrm{NC}=111$; if it meets none, $\mathrm{NC}=000$. In addition, these threshold criteria have been presented in the form of a weighted $\beta_{\mathrm{c}}$ factor that reflects the AGN-or SBN-dominance of the source. The results of such radio qualifications will be compared to the optical classification of the nuclear emission.

Our sample of sources is not complete across the whole $L_{\mathrm{FIR}}$ range due to the incompleteness of the redshift surveys at the time of the searches for $\mathrm{OH}$ among FIR galaxies. The sample is representative of the $\mathrm{OH}$ Megamaser population and the ULIRG at FIR luminosities above $10^{11.3} L_{\odot}$. The sample consists of a total of 51 sources, 35 of which are confirmed $\mathrm{OH}$ Megamasers. An additional five have still unconfirmed $\mathrm{OH}-\mathrm{MM}$ activity and a further 8 sources were claimed $\mathrm{OH}-\mathrm{MM}$ at one time but have been found not to be OH-MM. Three $\mathrm{OH}$ absorbers in the sample have a significant column density towards their nuclei, but do not have the right FIR pumping environment to have maser action. All sources in the sample have been selected on the basis of their FIR properties and luminosities and may serve adequately as a coherent but incomplete sample.

The data in Table B. 2 covers a total of 56 source components from 51 sources. Four sources have more than one component in the table; IR01364 has $4.83 \mathrm{GHz}$ and $8.44 \mathrm{GHz}$ data. The sources in Table B.1 are reorganized in Table B.2, in order to separate the $\mathrm{OH}$ Megamasers and the candidate sources with "unconfirmed" Megamaser activity with MMC designation in Col. 13 of Table B.1 (Klöckner 2004), and some sources without any OH-MM activity. This group of sources will be plotted with crosses rather than squares in the Figs. 1-4.

The FIR spectral index between 25 and $60 \mu \mathrm{m}$ has also been considered as a possible indicator (see de Grijp et al. 1985). Because of the strict FIR selection criteria of the sources in our $\mathrm{OH}-\mathrm{MM}$ sample, this parameter was not found to be a distinguishing one for the available data for this sample.

\subsection{The FIR- radio relation}

The relation between the nuclear radio luminosity and the FIR luminosity for $1.43 \mathrm{GHz}$ and $4.85 \mathrm{GHz}$ is displayed in Fig. 1. Early discussions of this relation at $1.4 \mathrm{GHz}$ presented values close to unity for the exponent (see Helou et al. 1985). This has been confirmed again recently for a much larger IRAS sample at $1.4 \mathrm{GHz}$, where the exponent is found to be 0.99 (Yun et al. 2001). Evaluation of this relation at $4.8 \mathrm{GHz}$ suggested an exponent of 1.26 at higher $L_{\mathrm{FIR}}$ (Wunderlich et al. 1987) or 1.07 for Megamaser galaxies (Henkel \& Wilson 1990). A recent evaluation of lower resolution data at 1.4 and $4.8 \mathrm{GHz}$ for a sample of ULIRGs with luminosity $\geq 10^{11.4} L_{\odot}$ suggests an exponent of 1.13 at $4.8 \mathrm{GHz}$ (Crawford et al. 1996).

Our luminosity range of $10^{10.6}$ to $10^{12.5} L_{\odot}$ is smaller than the five orders of larger samples (Price \& Duric 1992; Yun et al. 2001). With the exclusion of the three radio-loud Seyfert sources, IR02483+4302, IR13451+1232 and IR14566-1629, the main body of data points in Fig. 2 gives a relation of $L_{4.8 \mathrm{GHz}} \propto L_{\mathrm{FIR}}^{1.30 \pm 0.29}$, while at $1.4 \mathrm{GHz}$ the exponent is $1.20 \pm$ 0.29 . The three radio-loud sources stand out because of their

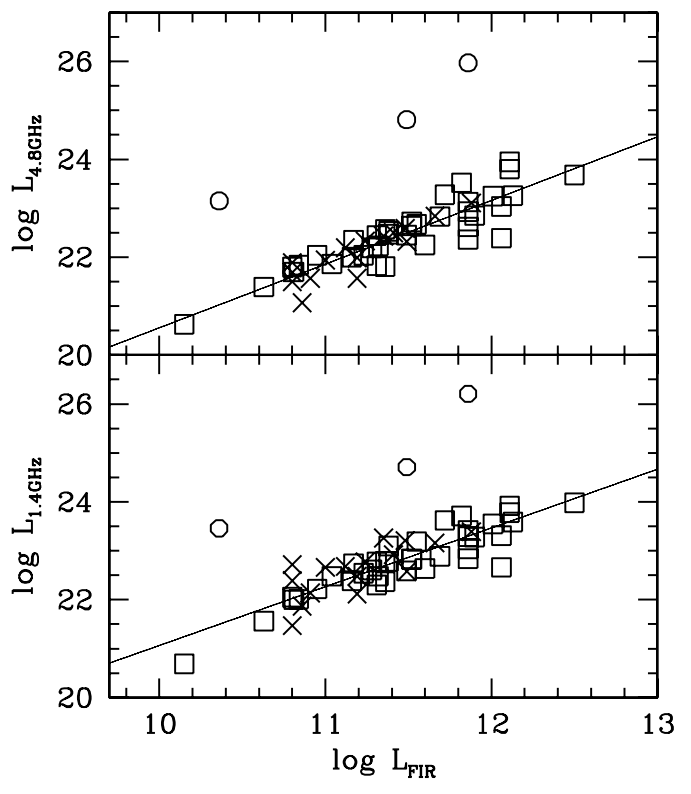

Fig. 1. The infrared-radio luminosity relation: a) the radio luminosity from the $4.8 \mathrm{GHz}$ data, b) the radio luminosity from the $1.4 \mathrm{GHz}$ data. The linear fit in the top frame gives a dependence of $L_{4.85 \mathrm{GHz}}=$ $L_{\mathrm{FIR}}^{1.30 \pm 0.29}$, while the exponent in bottom frame for the $1.4 \mathrm{GHz}$ data is $1.20 \pm 0.29$. The known Megamasers are designated with open squares. Unconfirmed sources and $\mathrm{OH}$ absorbers (see Table B.2) are given by crosses. The radio loud sources have been marked as circles and are excluded in the fits. The errors of the data points lie within the size of the individual markers.

high brightness temperatures and their radio luminosity, but they do not distinguish themselves in their spectral index. Our high-resolution data give exponents that suggest higher values than the unity slope values published earlier. Steepening of the relation at the highest luminosities is consistent with the results from other studies. There is also some evidence that the dispersion in the source distribution gets larger at higher luminosities, because of increasing contributions of compact radio sources and AGNs at higher luminosity (see Yun et al. 2001).

\subsection{The FIR-radio q-ratio}

The FIR-Radio ratio or $q$-ratio has been defined to express the efficiency of a galaxy in producing FIR flux as compared to the (mostly) nuclear radio continuum (Helou et al. 1985). Starburst galaxies emit a large fraction of their radiative energy in the FIR, while producing relatively little emission in the radio. A $60 \mu \mathrm{m}$ flux-limited ( $\geq 1.2 \mathrm{Jy}$ ) sample of starbursts shows a median value for $\langle q(4.8)\rangle=2.75 \pm 0.03$ (Condon \& Broderick 1991), while normal galaxies with distributed starbursts have $\langle q(4.8)\rangle=2.64 \pm 0.01$ (Condon et al. 1991a). The more extensive IRAS Bright Galaxy Sample (BGS) shows a narrow distribution centered on $\langle q(1.4)\rangle=2.34$, while the sources with AGNs span a wide range of $q$-ratios (CHYT; Yun et al. 2001). The pure AGNs in radio-selected samples generally have $q<$ 2 , but the $q$-ratios of the ultra-luminous BGS galaxies are in the starburst range or slightly higher (Condon \& Broderick 1988; Wilson 1988). VLA D-array studies (Condon et al. 1991a) have classified all sources with $q(4.8)>2.2$ as starbursts and all 


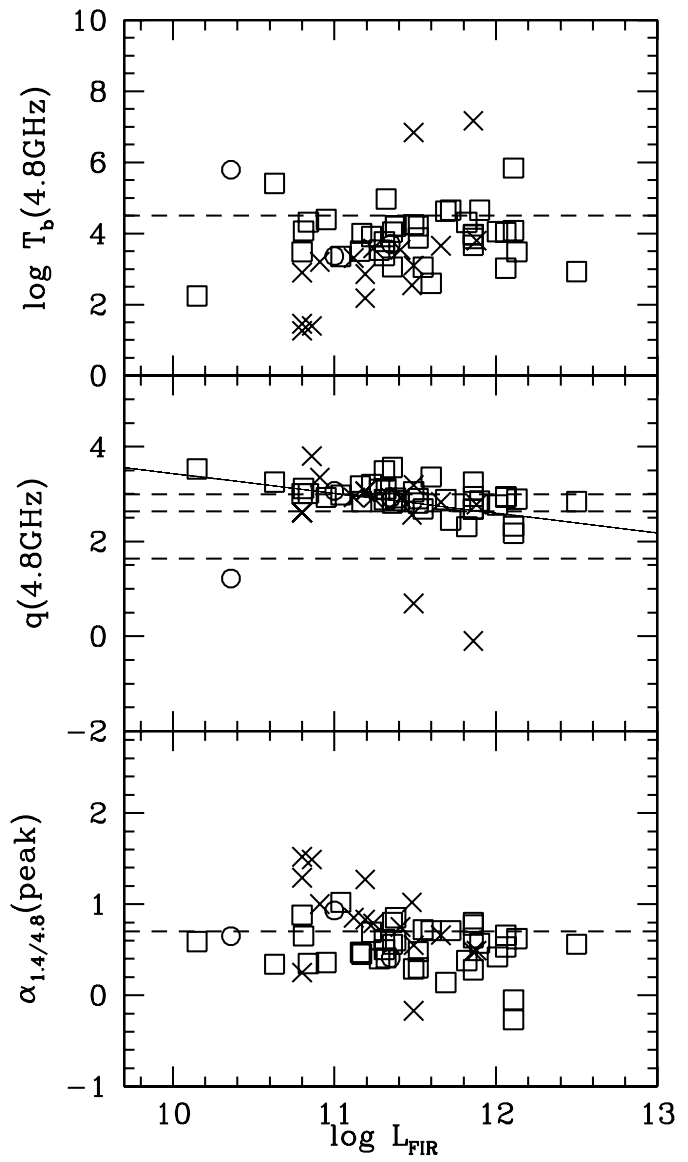

Fig. 2. The variation of the three main diagnostic parameters versus the FIR luminosity: $T_{\mathrm{b}}(4.8 \mathrm{GHz})$, the $q$-ratio at $4.8 \mathrm{GHz}$, and the peak spectral index. Top panel: the dashed line represents the value for $T_{\mathrm{b}}=4.5$ above which AGNs would occur (Condon et al. 1992). Middle panel: the fit in the middle panel gives a $q-L_{\mathrm{FIR}}$ dependence of $-0.29 \pm 0.29$. The dashed lines represent the threshold for radioloudness $q<1.64$, the mean value of $q(4.8)=2.64$ for galaxies with starbursts (Condon et al. 1991a), and the threshold for FIR-excess $q>$ 3 (Yun et al. 2001). Bottom panel: the dashed line represents the mean spectral index of 0.7 for the BGS (CHYT). Data points are as in Fig. 1.

sources below this threshold as AGNs. The BGS sources with FIR luminosities similar to those for our OH Megamaser sample are often dominated by significantly smaller radio components than their minimum possible FIR source sizes (CHYT).

The values of $q$ for 1.43 and $4.85 \mathrm{GHz}$ (see Table B.2, Cols. 8 and 9) have been displayed in the middle panels of Figs. 2-4. Lower values of $q$ indicate increasingly significant contributions of the AGN to the total radio emission; indeed, the three radio-loud sources have significantly lower $q$-ratios and higher brightness temperatures. The median value of $q(4.8)$ for our sample is 2.90 , which is slightly higher than the values for other samples. This difference could be due to the composition of the sample and the slightly higher spatial resolution of our radio data resulting in missing flux from extended emission. The $q$-ratio at $4.8 \mathrm{GHz}$ decreases with radio and FIR luminosities respectively as $L_{4.8}^{-0.29 \pm 0.29}$ and $L_{\mathrm{FIR}}^{-0.31 \pm 0.21}$, which is a direct result of the observed $L_{4.8}-L_{\text {FIR }}$ slope of 1.30 in Fig. 1. Similarly $q(1.4)$ varies as $L_{1.4}^{-0.18 \pm 0.20}$. For sources with high-frequency data at $8.4 \mathrm{GHz}$, a value of $q$ based on a flux

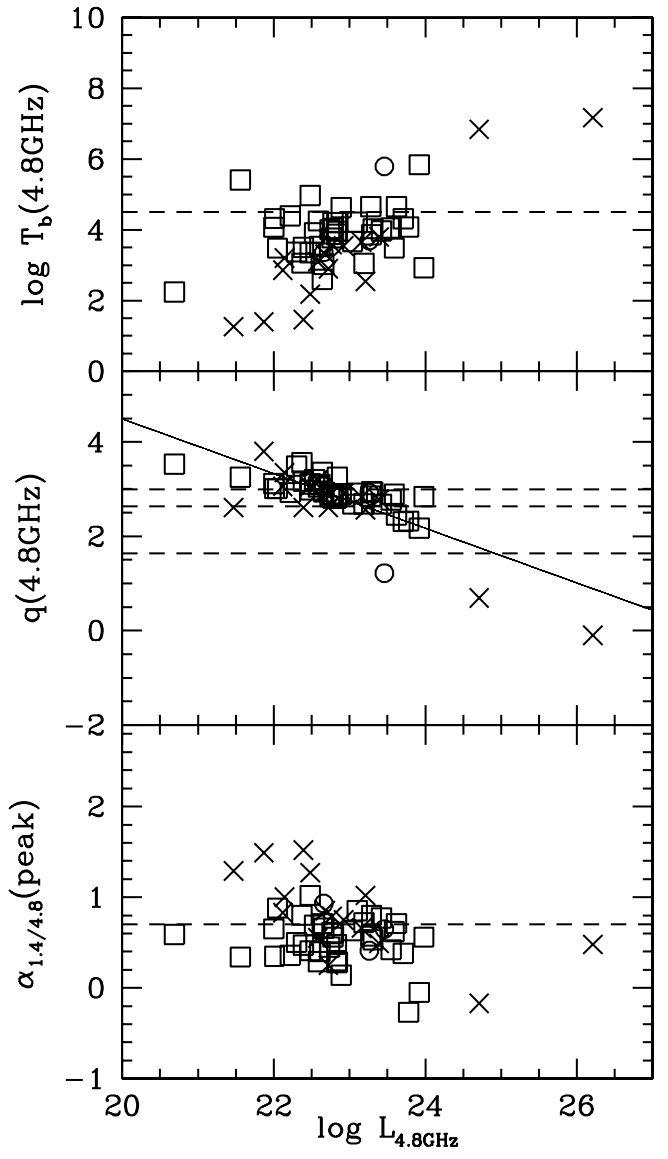

Fig. 3. The variation of the three main diagnostic parameters versus the $4.85 \mathrm{GHz}$ radio luminosity: $T_{\mathrm{b}}(4.8 \mathrm{GHz})$, the $q$-ratio at $4.8 \mathrm{GHz}$, and the peak spectral index. Top panel: the dashed line represents the value for $T_{\mathrm{b}}=4.5$ above which AGNs would occur (Condon et al. 1992). Middle panel: the fit in the middle panel gives a $q-L_{4.85} \mathrm{GHz}$ dependence of $-0.31 \pm 0.21$. The dashed lines represent the threshold for radio-loudness $q<1.64$, the mean value of $q(4.8)=2.64$ for galaxies with starbursts (Condon et al. 1991a), and the threshold for FIR-excess $q>3$ (Yun et al. 2001). Bottom panel: the dashed line represents the mean spectral index of 0.7 for the BGS (CHYT). Data points are as in Fig. 1.

interpolation from $8.4 \mathrm{GHz}$ to $4.85 \mathrm{GHz}$ is used in the evaluation. Also the sources with multiple components have one common $q(4.8)$ value based on the integrated $4.83 \mathrm{GHz}$ flux. We note that the dispersion of the data points increases towards higher FIR luminosities, which results from the increased dispersion seen in Fig. 1.

In classifying our sample sources at higher radio resolution, we consider that the mean value for our sample is higher than for other samples at $q(4.8)=2.90$. Therefore, we have designated all galactic nuclei with a luminosity of less than $40 \%$ of the mean to be an AGN or all sources with $q(4.8)<2.50$ have a unit second digit in NC. The $q(4.8)$ values for our VLA A-array data are typically $10-20 \%$ larger than the $q(1.4)$ values.

\subsection{The brightness temperature}

The brightness temperature of the sources has been calculated for both the $1.43 \mathrm{GHz}$ and $4.85 \mathrm{GHz}$ data (Table B.2, Cols. 6 


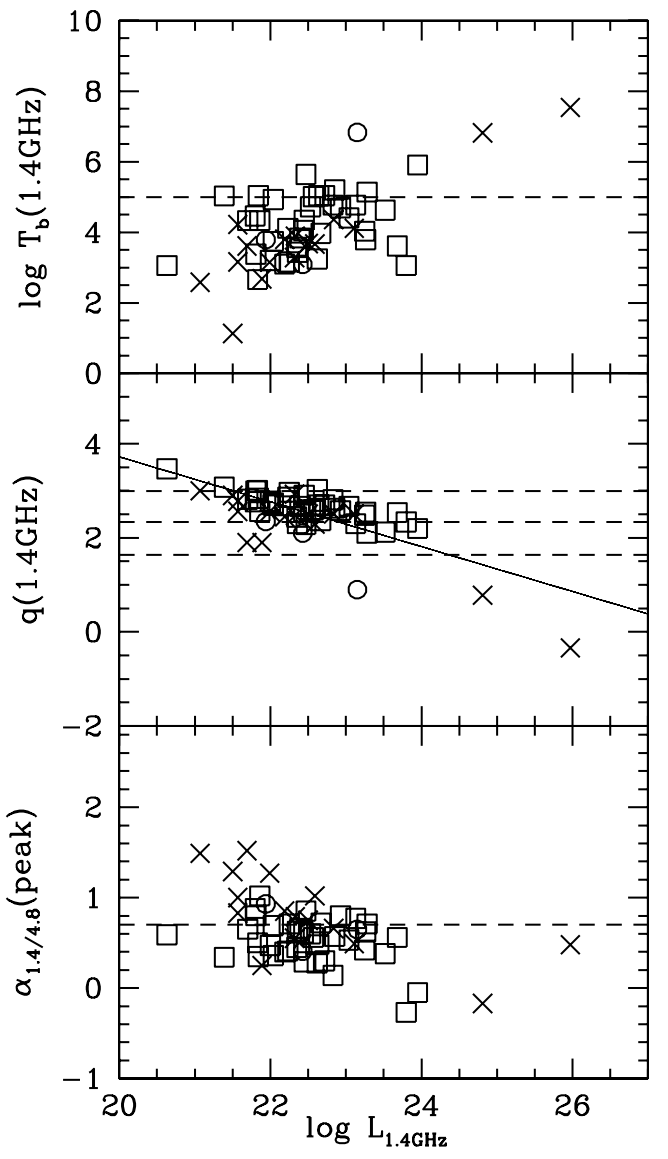

Fig. 4. The variation of the three main diagnostic parameters versus the $1.43 \mathrm{GHz}$ radio luminosity: $T_{\mathrm{b}}(1.4 \mathrm{GHz})$, the $q$-ratio at $1.4 \mathrm{GHz}$, and peak the spectral index. Top panel: the dashed line represents the value for $T_{\mathrm{b}}=5.0$ above which AGNs would occur (Condon et al. 1992). Middle panel: the fit in the middle panel give a $q-L_{1.4 \mathrm{GHz}}$ dependence of $-0.31 \pm 0.21$. The dashed lines represent the threshold for radio-loudness $q<1.64$, the mean value of $q(4.8)=2.34$ for the BGS, and the threshold for FIR-excess $q>3$ (Yun et al. 2001). Bottom panel: the dashed line represents the mean spectral index of 0.7 for the BGS (CHYT). Data points are as in Fig. 1.

and 7) and displayed in the upper panels of Figs. 2-4. The uncertainty in the brightness temperature is presented in Table B.2 and are smaller than the plotted symbols. The higher resolution of the $C$-band data renders the $4.85 \mathrm{GHz}$ brightness temperature a better indicator of compact sources. Furthermore, the compact luminous FIR sources become optically thick for freefree absorption at about $3 \mathrm{GHz}(\mathrm{CHYT})$. As a result the spectrum may flatten in this region and no starburst can be brighter than $T_{\mathrm{b}} \approx 10^{5} \mathrm{~K}$ above $1 \mathrm{GHz}$ or brighter than $10^{4.5} \mathrm{~K}$ above 4.85 GHz (see Condon et al. 1992).

Most sources in our sample have $T_{\mathrm{b}}(4.85)<10^{4.5} \mathrm{~K}$, while only the three radio-loud Seyferts have $T_{\mathrm{b}}(4.85)>10^{5.8} \mathrm{~K}$. For the purpose of this classification, we suggest that all galaxies with $\log T_{\mathrm{b}}(4.8) \geq 4.5$ or $\log T_{\mathrm{b}}(1.4) \geq 5.0$ tend towards AGN dominance (Condon et al. 1992). The brightness temperature for sources with $8.44 \mathrm{GHz}$ data has not been converted to $4.85 \mathrm{GHz}$. Galaxies with temperatures above this threshold have a unit as the first digit of the nuclear code NC.
The three diagrams for the brightness temperature show a slight increase of $T_{\mathrm{b}}$ with increasing luminosity, because the brightness temperature is indeed proportional to the flux density. This observed increase could result from more prominent contributions from AGNs and compact/intense starbursts.

\subsection{The spectral index}

The third nuclear indicator is the radio spectral index for the integrated and peak flux densities, which have been given in Table B.2, Cols. 10 and 11. The spectral indices based on the peak fluxes have been displayed in the bottom panels of Figs. $2-4$. In general, it is found that $\alpha \approx 0.75$ for a pure synchrotron spectrum, while $\alpha \approx 0.1$ for a pure thermal spectrum. Flux-limited samples of extra-galactic radio sources (nearly all powered by AGNs) suggest that relatively flat radio spectra $(\alpha \leq 0.5)$ are dominated by synchrotron self-absorption. For these sources the brightness temperatures are comparable to the kinetic temperature of the relativistic electrons producing the synchrotron radiation. Recent results show that some of such flat spectrum sources are resolved at higher frequencies and have brightness temperatures close to only $10^{4} \mathrm{~K}$, which is still too low to be explained with synchrotron self-absorption. Lower brightness temperatures are consistent with free-free absorption of optically thin synchrotron radiation, plus a smaller component of thermal emission by $\left(T_{\mathrm{b}} \approx 10^{4} \mathrm{~K}\right)$ thermal electrons in HII regions. Therefore, moderately flat $(\alpha \leq 0.5)$ radio spectra by themselves cannot be used to rule out starbursts among the luminous FIR galaxies (see Condon et al. 1991a). However, steeper spectra do indicate significant starburst contributions. The median spectral index of normal galaxies and extended starbursts is $\langle\alpha\rangle \approx 0.75$ (Gioia et al. 1982; Condon 1983), which is characteristic of synchrotron radiation. Most of the IRAS BGS sources have comparably steep $(\alpha \approx 0.7)$ spectra between 1.43 and $8.44 \mathrm{GHz}$ (CHYT).

In this classification, all sources with peak spectral index $\alpha_{4.85}^{1.43} \leq 0.4$ are classified as AGN (third unit in NC). We also note that the bottom panels of Figs. 2-4 may indicate that the higher FIR and radio luminosity sources have flatter radio spectra. Crawford et al. (1996) also noted this tendency at higher FIR luminosities. While any dependence in this data sample is not significant, such dependence could indeed indicate that the radio AGN-domination becomes more prominent at higher frequencies.

\subsection{Redshift dependence}

The relation between the integrated FIR luminosity and the radio luminosity of a partially or unresolved nuclear core could still be affected by the redshift dependent spatial resolution. Sources at larger distance become less resolved in the radio and have relatively larger integrated flux densities. For the highest redshift source in our sample the beam size corresponds to a physical size of $1.7 \mathrm{kpc}$. Because the IRAS FIR data would be less influenced in this manner, the $q$-values for more distant sources could become smaller. Given the selection criteria of the $\mathrm{OH}$ Megamaser searches, the current sample is flux-limited 
in both the radio and the FIR, and is not complete at higher redshift with a selection bias against weak radio sources at $z \geq 0.1$.

The current sample does not show any significant evidence that the $L_{4.8 \mathrm{GHz}}-L_{\mathrm{FIR}}$ relation and the other parameters discussed here are affected by a redshift dependence. In particular, the redshift would not affect the classification of the nuclear sources.

\section{Analysis of radio data}

The radio characteristics of the total sample of 51 sources in Table B. 2 show that a majority is fuelled by starbursts. Eleven sources, or $21.6 \%$ of the sample, are found to have double or multiple components at $1.43 \mathrm{GHz}$ or at $4.85 \mathrm{GHz}$. For three of these sources, the $1.43 \mathrm{GH}$ components do not have detected $4.85 \mathrm{GHz}$ counterparts.

\subsection{Radio diagnostics in $2 D$}

The availability of three independent radio parameters makes it possible to construct diagnostic diagrams to visualize the radio classification. In the three diagrams of Fig. 5, the $q$-ratio, the brightness temperature, and the peak spectral index have been plotted against each other. The thresholds used for our classification have been added to the diagrams (dashed lines) to indicate where in the above discussions the boundaries between AGN- and SBN-dominated sources have been chosen. Clearly a large fraction of the sources falls close together in the region of the diagrams classified as part of a single SBN-dominated population. The classification of the sources in these transition regions depends strongly on the threshold values chosen for the $q$-ratio at $4.8 \mathrm{GHz}, \log T_{\mathrm{b}}(4.8 \mathrm{GHz})$, and the peak $\alpha_{4.85}^{1.43}$. We find that further fine-tuning of the threshold values in order to optimize the classification will only shift the balance for the boundary cases, but would not affect the general conclusions of this paper.

Particularly the $q(4.8)-T_{\mathrm{b}}$ and the $\alpha_{4.85}^{1.43}-T_{\mathrm{b}}$ diagrams do exhibit a continuous distribution of points going from AGN-dominated sources to SBN-dominated. This could be interpreted as a mixing curve that describes the variation of the diagnostic parameters as the dominant radio contributor changes. The dotted lines illustrate the approximate halfway point between the SBN and AGN areas along this mixing line in Fig. 5.

\subsection{Radio diagnostics in $3 D$}

The diagrams in Fig. 5 are three 2-D projections of the 3-D sample distribution. Suggestions have been made to indicate the boundaries between AGN and SBN by means of the dotted line in each of the diagrams. These dotted lines define a 3-D surface that goes through the 3-D reference point $(q, T$, $\alpha)=(2.5,4,0.5)$, which lies just inside the tip of the 3 -D box formed by the $1-\mathrm{D}$ criteria (dashed lines). The other two points used for defining the 3-D surface are $(4,6,0.5)$ and $(1,4,1)$. The distance of a data point $(q, T, \alpha)$ to this 3-D surface serves as a measure of the AGN-domination or SBN-domination of

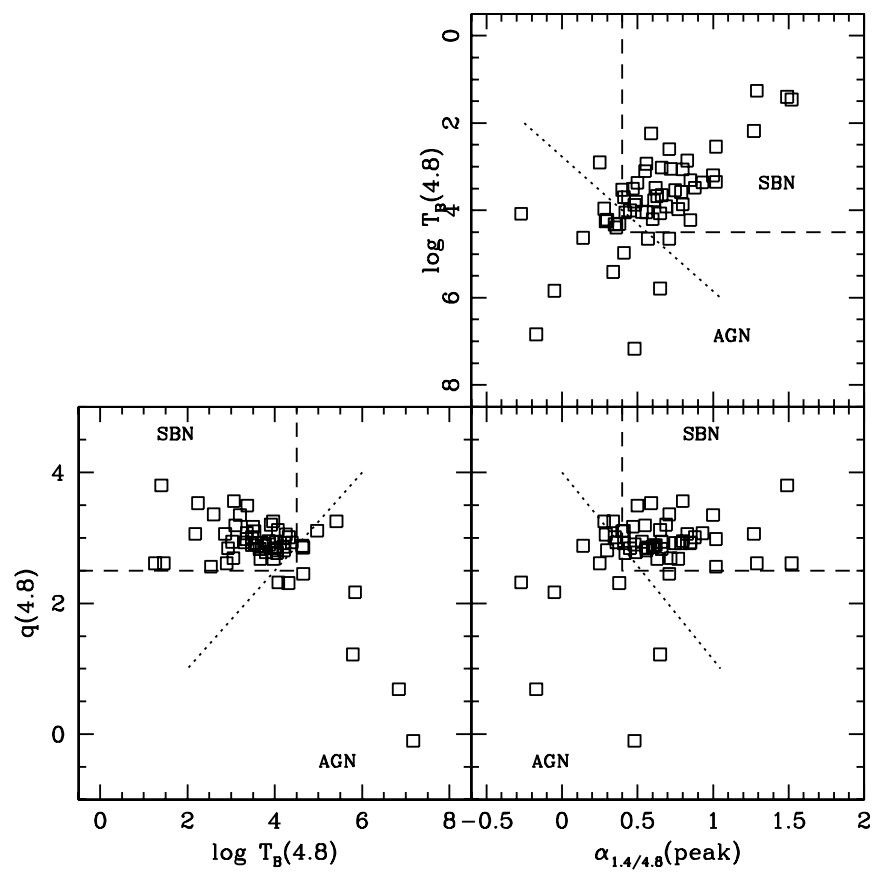

Fig. 5. Radio diagnostic diagrams. The $q$-ratio, the brightness temperature $T_{\mathrm{b}}$ for the $4.85 \mathrm{GHz}$ data, and the peak spectral index between $1.43 \mathrm{GHz}$ and $4.85 \mathrm{GHz}$ have been plotted against each other. The dashed lines in the frames represent the classification criteria used in this paper and form the quadrants within which AGNs and SBNs are located. These dashed lines form a 3-D box containing the SBN population. Data points are as in Fig. 1. The dotted lines in the three diagrams represent the boundary between the AGN and SBN classifications. These lines are the three projection lines of a 3-D surface going through the $(q, T, \alpha)=(2.5,4,0.5)$ point in this surface, which lies just inside the corner point of the 3-D box given by the dashed lines. The distance of data points to this 3-D surface and the three dotted lines is representative of the AGN/SBN classification of the points.

the source, which is described as:

$\beta_{c}=0.308\left[q(4.8)-0.75 * T_{\mathrm{b}}(4.8)+3 * \alpha-1\right]$,

where the equation between brackets (set equal to 0) actually defines the 3-D surface itself. SBN-like sources have positive values for this activity factor $\beta_{\mathrm{c}}$ and AGN-like sources have negative values. The values of $\beta_{\mathrm{c}}$ have been presented to Table B. 2 in Col. 13. We note that the zero-point for our bestguess $\beta_{\mathrm{c}}$ scale may not be exact and may need to be shifted.

\subsection{Qualification with nuclear code}

For the total sample there are only two sources with three AGN units: the radio-loud source IR02483+4302 and the well-known Seyfert 1 galaxy IR12540+5708 (Mrk 231). There are nine sources with two AGN units, including IR13451+1232 and the radio-loud OH-absorber IR14566-1629. Another eight sources have one AGN unit. There are 32 sources without any AGN units.

For the 35 confirmed Megamaser sources, we find that $20(57.1 \%)$ sources have no AGN units, seven (20\%) have one unit, seven (20\%) have 2 units, and one (2.9\%) has three units. 
Eight nuclear sources show strong AGN activity with two or three AGN units comprising $22.9 \%$ of the OH-MM sample. The 27 sources with 0 or 1 AGN units are SBN-dominated and they account for $77 \%$ of the whole sample. In the extreme case, assigning AGN status to all sources with one or more units gives a division of $43 \%$ AGN-dominated and $57 \%$ SBN-dominated.

The sample of 16 sources with unconfirmed or no $\mathrm{OH}-\mathrm{MM}$ emission has relatively more $\mathrm{SBN}$-dominated sources: twelve sources $(75 \%)$ have no units, there is one $(6.3 \%)$ with one unit, two $(12.5 \%)$ with two units, and one $(6.3 \%)$ with three. This non-Megamaser (comparison) group has even more $(81) \% \mathrm{SBN}$-dominated sources than the "confirmed" Megamaser group. Because these differences are not very significant and could be related to selection effects, we cannot draw any conclusion from them.

\subsection{Qualification with $\beta_{c}$}

The activity factor $\beta_{\mathrm{c}}$ represents a weighted qualification of the three diagnostic parameters with uneven weights, unlike the case of the digit method described above. Using this classification on the sample of $35 \mathrm{MM}$ sources, there are $11(31.4 \%)$ sources with $\beta_{\mathrm{c}} \leq 0$. Of the comparison sample with 16 sources with no MM emission, there are 3 sources $(18 \%)$ with $\beta_{\mathrm{c}} \leq 0$. These numbers are consistent with assigning AGN activity to MM sources with 2 or $3 \mathrm{NC}$ units and some of the sources with one unit. For the non-MM sample the 3 sources with 2 or 3 units also have $\beta_{\mathrm{c}} \leq 0$. A comparison of the two methods in Table B. 2 shows that there is a clear correlation between the $\mathrm{NC}$ numbers and $\beta_{\mathrm{c}}$. Considering that the one unit $\mathrm{NC}$ sources form a transition group, the $\beta_{\mathrm{c}}$ activity factor is more discriminatory for sources close to the boundary. The $\beta_{\mathrm{c}}$ value could be considered as an overall radio classification with the caveat that the zero value of the $\beta_{\mathrm{c}}$ scale may shift with the choice for the 3-D surface, that forms the half-way point between AGNand SBN-domination.

\section{Evaluation of the radio classification}

The radio emission from the nuclear region has been interpreted as a superposition of varying contributions from nuclear and circum-nuclear starbursts, and from an active galactic nucleus. Similarly, the FIR emission from Seyferts has been explained as a composite of thermal and non-thermal emissions from the active nucleus, thermal emission from a starburst, and cool thermal emission from the disk (Miley et al. 1985; Ward et al. 1987; Mazzarella et al. 1991; Heckman 1998). Particularly, a spectral analysis of optical and radio emissions recognizes the dominance of the various emission components resulting from AGN and starburst activities. The classification in this discussion represents the relative energetic dominance of an AGN or an SBN in the radio.

A strongly AGN-dominated radio continuum can easily be recognized from the $L_{4.8}-L_{\mathrm{FIR}}$ diagram, where three radio-loud sources are indeed classified as AGNs. Earlier studies identified such radio-loud sources as galaxies having a linear (double, triple or jet-like) structure in the nucleus (Wilson 1988), but with the radio and FIR emission dominated by a Seyfert nucleus. Linear (elongated) structures have indeed been seen in the maps for the radio-loud sources, IR13451+1232 and IR14566-1629 (not shown in Fig. A.1). However, five sources (IR05414+5840, IR11069+2711, IR16399-0937, IR16577+5900, IR17526+3253) in our sample show linear extensions that are related to starburst activity in the inner disk of the galaxy.

The 1-D nuclear code NC and the activity factor $\beta_{\mathrm{c}}$ have been used to classify the radio properties of the sample sources. Comparisons with optical classifications have been used to arrive at a classification for the energetic dominance of the nucleus. This classification $\mathrm{A}(\mathrm{GN})$ or $\mathrm{S}(\mathrm{BN})$ has been entered in Col. 15 of Table B.2. This classification designates twelve $(34 \%)$ of the 35 OH-MM sources and three (19\%) of the 16 non-OH-MM as AGNs. The present sample has $33 \%$ AGN-like sources $\left(\beta_{\mathrm{c}} \leq 0\right)$ for $\log L_{\mathrm{FIR}} \geq 11.75$, while there are $21 \%$ in the range below 11.75 . This suggests that the AGN contribution to the radio becomes more prominent at higher FIR luminosities, which is also supported by some earlier studies (Sanders et al. 1988; Crawford et al. 1996). The optical studies also suggest that the AGN contributions become more dominant at higher FIR luminosity (Sanders et al. 1988; Baan et al. 1998; Veilleux et al. 1995). This increase of frequency of AGN with increasing FIR and radio luminosity could account for any increased dispersion in the $L($ FIR $)-L$ (radio) relation (Fig. 1; Sect. 3.1.1) at higher luminosities. An increase in FIR luminosity may relate to the increased concentration of nuclear material, such that the AGN contribution to the radio suffers increasingly from free-free absorption. Because the $10 \%$ frequency of radio-loud sources among the AGN may not vary with FIR luminosity (Yun et al. 2001), there will be higher fraction of non-radio-loud AGN sources at higher luminosities.

The diagrams show that the number of sources with large $q$-ratios is larger than that found in other samples. The much larger BGS radio sample (Yun et al. 2001) at lower spatial radio resolution shows an FIR-excess with $q(1.4)>3$ in only $0.6 \%$ sources. In our sample an FIR-excess is found for six sources $(11.7 \%)$ at $1.4 \mathrm{GHz}$ and for 19 sources $(37.2 \%)$ at $4.8 \mathrm{GHz}$. Among the MM sample alone some $40 \%$ of sources (14) show FIR-excess; the percentage is only $14 \%$ among non-MM sources. The sources with FIR-excess are found predominantly at lower luminosity because the FIR-excess threshold has been configured as a constant rather than a sloped line positioned above the fitted curves in Fig. 1. The much higher percentage of FIR-excess sources among the MM sample reflects on the FIR pumping of the $\mathrm{OH}$ in this population of warm and (ultra-)luminous FIR sources.

Similarly Yun et al. (2001) find $1.3 \%$ of the BGS sources to have a radio-excess with $q<1.64$. Our sample shows radio-excess only for our three radio-loud non-MM sources (IR02483+4302, IR13451+1232, and IR14566-1629), which represents nearly $6 \%$. The boundaries for FIR-excess and radio-excess have been indicated in the figures.

High-resolution VLBI studies of Seyfert galaxies provide evidence that high brightness components may indeed be present in many of these galactic nuclei. A study by Roy et al. (1994) shows that compact cores are more common in Seyfert 2 
galaxies (46\%) than in Seyfert 1 galaxies (26\%). Increasing the free-free absorption optical depths in the Narrow Line Region may account for the different detection rates within a unified AGN model. Similarly, Lonsdale et al. (1993) find that more than half their sample of luminous FIR galaxies shows compact high-brightness $\left(T_{\mathrm{b}}>10^{5} \mathrm{~K}\right)$ components on scales of 5-150 mas, with a mean of only twelve percent of the integrated flux density of the galaxy. Luminous FIR galaxies from our sample could have weak dust-obscured compact nuclear sources, but it is not clear whether such embedded sources would affect a radio classification significantly.

\subsection{Comparison with optical classification}

The division between SBN-dominance and AGN-dominance is different for the radio and optical spectral analysis. An optical study for approximately the same sample of $\mathrm{OH}$ Megamaser sources shows that the majority of sources show signs of optical AGN activity (Baan et al. 1998). Of the classifiable optical sources, a total of $51 \%$ can be classified as Sy 1 and 2, while only 23\% are SBNs. The remaining 26\% are Composite sources with a mixture of SBN and AGN characteristics. Therefore, a total of $77 \%$ of the optical sample shows some AGN-dominant characteristics. The above discussion shows that only $27 \%$ of the radio sources have dominant or strong AGN characteristics with $\beta_{\mathrm{c}} \leq 0$. The classification procedures of the nuclear activity in the radio and the optical do not operate on the same characteristics of the underlying energy source, and do not produce a general one-to-one correlation between the radio and optical classifications.

The disparity of the radio and optical classifications for this sample can be seen in the Cols. 12 and 13 versus Col. 14 in Table B.2. Figure 6 displays a comparison of $\beta_{\mathrm{c}}$ with the standard optical diagnostic ratios $[\mathrm{OI}] / \mathrm{H} \alpha$ and $[\mathrm{NII}] / \mathrm{H} \alpha$, which were used for the optical classification of the sample sources (Baan et al. 1998). Sources with more extreme pos/neg values of $\beta_{\mathrm{c}}$ have also similar optical classifications. For the data points in between, we find only a weak correlation. For smaller values of $\beta_{\mathrm{c}}$, we find sources with a large spread of optical ratios and with subsequent classifications ranging from HII (on the left of each diagram) to Seyfert (on the right). Radio starbursts within this sample cover a wide range of optical characteristics between pure Seyfert and pure starburst.

It has been suggested that shocks and an intense radiation field in the extreme environment of compact nuclear starbursts could sufficiently modify the optical diagnostics of these nuclei to make them mimic LINERs and Composite Spectrum Nuclei (CSN) (see Baan et al. 1998). Similarly, the NIR classification using ISO data suggests that the majority of the ULIRGs are SBN-dominated (Lutz et al. 1998; Genzel et al. 1998). For these sources the observed emission lines indicate starburst activity, while there are no high ionization lines (the signature of an AGN) present. We find that the classification of the overlapping sources in the NIR sample and ours agree strongly.

A comparison of optical and radio classifications reveals some disagreement in $44 \%$ (15 out of 41) of the optical

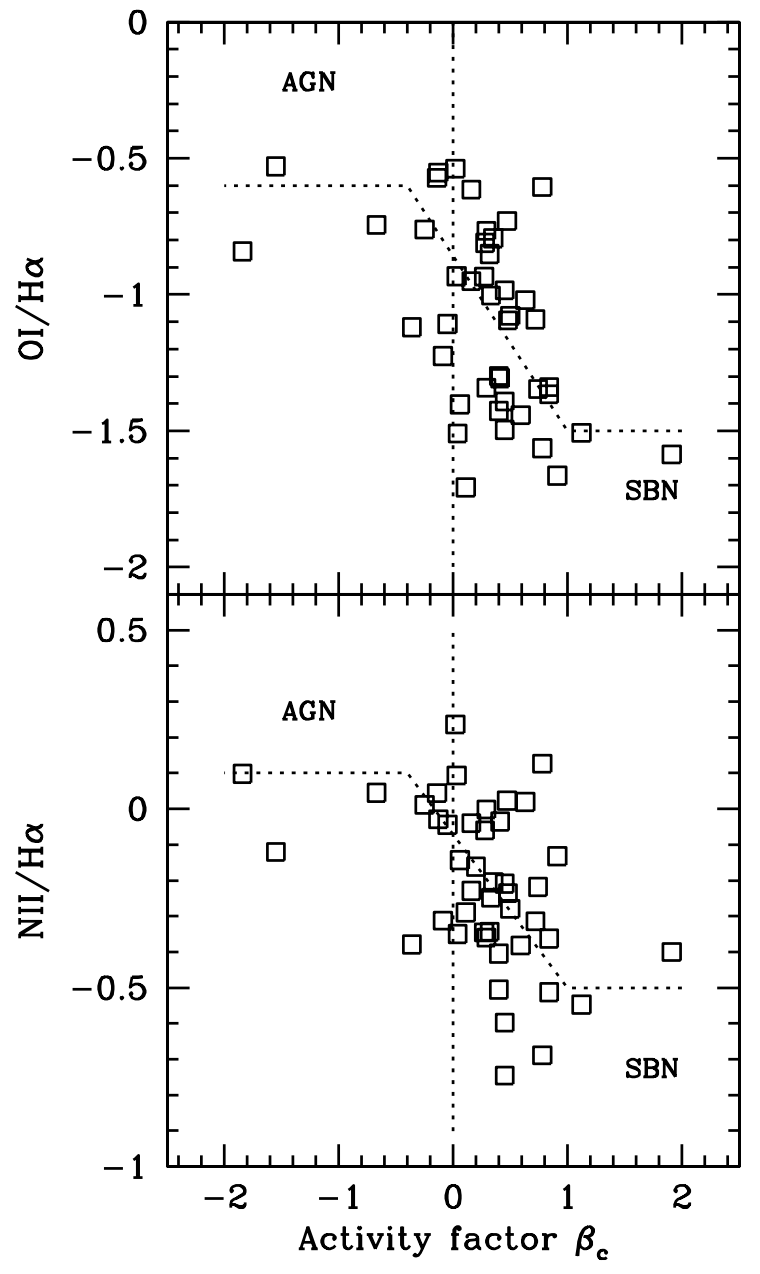

Fig. 6. Radio classification versus optical diagnostic line ratios. The optical diagnostic line ratios have been compared with the activity factor $\beta_{\mathrm{c}}$ for the sources in both samples. The dotted line is a suggested trend in the data points from pure (radio and optical) SBN to pure AGN.

classified sources. For this discussion we will take the radio classification as a more accurate representation of the properties of the nuclear region than the optical line ratios. Many of the optical/radio discrepancies would disappear, if at least the twelve optical LINERs and CSNs without radio AGN could be reclassified as SBN-dominated on the basis of their radio properties as given in Table B.2 Col. 14. Two sources have radio AGN characteristics (IR13126+2452 and IR15107+0724) but have been classified as an optical SBN; here a radio AGN could be buried within an optically dominating circum-nuclear SB. Any (re-)classifications have been incorporated in Col. 15 of Table B.2.

There are also four optical Seyferts that have zero (3 sources) or one (Arp 220) NC unit and positive $\beta_{\mathrm{c}}$. Arp 220 has been classified optically as a LINER (Vielleux et al. 1999) or even a Seyfert (Baan et al. 1998). ISO studies show that Arp 220 is a ULIRG that is clearly fueled by a nuclear starburst (Genzel et al. 1998). Global VLBI studies confirm the absence of a dominant high-brightness compact radio source and show a cluster of supernova remnants distributed over about 
100 mas (35 pc) (Smith et al. 1998). These extreme starbursts may mimic optical emission line ratios typical of Seyferts.

\subsection{The superposition of SBNs and AGNs}

Many (ultra-) luminous FIR sources show radio SBN characteristics, while optically they tend towards Seyfert and LINER characteristics. This lack of direct correlation suggests that many of the high-luminosity sources would have an AGN at varying stages of activity in combination with a circumnuclear starburst. However, this combination cannot easily explain all observed discrepancies between optical and radio characteristics (CHYT; Baan et al. 1998). A simple (linear) mixing curve between AGN-dominated and SBN-dominated sources has been used to explain the NIR characteristics of ULIRGs (Genzel et al. 1998). Similarly, the Megamaser sample shows a well-behaved sequence in the optical line-ratio diagrams running from SBN to LINER to Seyfert (see Fig. 11 of Baan et al. 1998). However, purely linear mixing between AGN and SBN contributions does not explain satisfactorily the observed source distribution in the optical diagrams. Other (nonlinear) mixing schemes may be used for a better representation for the optical line ratios, and would indeed place the optical LINERs half-way between the optical SBNs and AGNs.

A radio $S B N-A G N$ mixing scenario could also be devised for the diagnostic parameter spaces of Fig. 5. The 3-D surface described in Sect. 3.4.2 suggests a separation between AGN and SBN dominated regimes, which divides a continuous distribution in two regions. Again, a non-linear mixing procedure between radio-FIR SBNs and AGNs could represent this general trend, and could put the halfway point close to the $\beta_{\mathrm{c}}$ surface. The clumping of SBN sources close to this transition suggests a physical threshold for a transition between the two groups, which may be related to the relative absence of a dusty environment in AGN-dominated sources and the missing optical-to-FIR conversion.

\section{Conclusions}

The radio and FIR characteristics of the nuclei of Megamaser galaxies are instrumental for understanding the activity at the nuclei of (ultra-) luminous FIR galaxies. High-resolution structural and spectral information at $L$-and $C$-bands have been used to classify the nature of the energy source for the radio emission of Megamaser nuclei as SBN-dominated or AGN-dominated.

The nature of the radio nuclei has been determined using the brightness temperature, the spectral index and the $q$-value as diagnostic indicators. Diagnostic diagrams (Fig. 5) using these parameters show a significant spread in parameter space and a clumping of SBN-diagnosed sources with very similar properties. Only the sources with identifiable AGN tendencies show discrepant values for the diagnostic parameters. As a first approach, we have used each of the three diagnostic parameters separately to form a Nuclear Code (NC), in order to determine the relative locations of sources within these diagnostic diagrams and to determine their radio nature. As a second approach we have used a weighted representation of these three parameters to define an activity factor $\beta_{\mathrm{c}}$ on a continuous scale for the determination of the AGN- and SBN dominance in the radio. This $\beta_{\mathrm{c}}$ value and the number of $\mathrm{NC}$ digits provide a consistent picture of the radio nature of the nuclei.

A total of $77 \%$ of the $35 \mathrm{OH}$ Megamaser sources in our radio sample have dominant SBN (0 or 1 units in NC) characteristics. Using the $\beta_{\mathrm{c}}$ value as a criterion this number is $68 \%$. For the 16 non-MM sources $78 \%$ have SBN-dominance using both $\beta_{\mathrm{c}}$ and the nuclear code. Some $63 \%$ of our total sample shows no sign at all of AGN activity in the radio. The prominence of SBN-dominated radio sources in our sample is significant and characterizes the nature of (U)LIG sample.

Of the three diagnostic parameters, we find that only the $q$-ratio reveals a clear underlying relation between the FIR and the radio luminosity. Our radio - FIR luminosity relation is slightly steeper than for other surveys because the radio apparently becomes more pronounced at higher luminosities for our strongly biased Megamaser sample. Also the brightness temperature $T_{\mathrm{b}}$ is found to increase with luminosity, but less steeply than with an anticipated linear dependence. This suggests that the contributions from AGNs and more compact and intense starbursts become more prominent at higher luminosities. Also at higher FIR and radio luminosity they have flatter radio spectra, which may suggest that the radio AGN-domination becomes more prominent at higher luminosities.

The current sample distinguishes itself from other surveys particularly in its extreme FIR properties. FIR-excess is found for six sources (11.7\%) at $1.4 \mathrm{GHz}$ and for 19 sources (37.2\%) at $4.8 \mathrm{GHz}$, as compared with the $0.6 \%$ for the much larger BGS radio sample (Yun et al. 2001) at lower spatial radio resolution. Among the MM sample alone some $40 \%$ of sources (14) show FIR-excess; the percentage is only $14 \%$ among non-MM sources. FIR-excess is found predominantly at lower luminosity because of the non-linear radio-FIR dependence. Of course, the higher FIR-excess percentage reflects on the FIR pumping for the $\mathrm{OH}-\mathrm{MM}$ activity among warm and (ultra-)luminous FIR sources and on the effective optical-to-FIR conversion in the extreme nuclear environment of these sources. On the other hand, radio-excess is not found among MM sources but only for three radio-loud non-MM sources (6\%), which is higher than the $1.3 \%$ of the BGS sources (Yun et al. 2001). There is no evidence for a dependence on redshift for the diagnostic parameters.

The classification of sources on the basis of their radio properties is clearly not in agreement with optical classification schemes of nearly the same sample, which find that the SBN-like - AGN-like division is 33\%-67\% (see Baan et al. 1998). A further $22 \%$ of the AGN-like optical sample shows composite spectra with both AGN and SBN characteristics. For some $18(44 \%)$ of the 41 optically classified sources there is a clear disagreement between the radio and optical classifications. Twelve optical LINERs and CSNs show no sign of radio AGN activity at all, and should actually be classified as SBNs. On the other hand, there are four optical Seyferts with zero (3) or one unit (Arp 220) radio sources. These sources would be SBNs, where the intense and compact nuclear starburst could mimic AGN characteristics with LINER, CSN, or even Seyfert optical line ratios. Two other MM sources have optical SBNs 
with small negative $\beta_{\mathrm{c}}$, which would be radio AGN buried inside a circum-nuclear starburst. After this reclassification there are twelve (34\%) AGNs among the OH-MM and three (19\%) among the non-OH-MM.

It has been investigated in the literature whether an intense nuclear starburst could mimic the presence of an AGN. The extreme shocked and ionizing environment in the nuclear region could indeed achieve this situation (see Baan et al. 1998, and reference therein). Another option is the co-existence of an AGN amidst a (circum-) nuclear starburst. The changes in the properties could then be related to mixing properties of the AGN and SBN components. This effect is confirmed by the continuum of sources with clearly AGN-dominated on one side (negative values $\beta_{\mathrm{c}}$ ) and SBN-dominated on the other side (positive values $\beta_{\mathrm{c}}$ ) in the diagnostic diagrams (Fig. 5). While this distribution does not explain our optical-radio discrepancies in detail, it may explain the general trends in both the radio and optical characteristics. Such a mixing scheme has been successfully used for the NIR results on prominent galaxies (Genzel et al. 1998). A systematic trend from HII sources to LINERs to Seyferts is also visible in the optical diagnostic diagrams (Fig. 11 in Baan et al. 1998; Fig. 6 in Veilleux et al. 1995). We have not been able to produce a physically plausible AGN-SBN mixing procedure for the optical and radio diagnostic diagrams. However, on the basis of our results it appears that SBN-dominated sources make up a large $(\approx 77 \%)$ fraction of the (U)LIRG population. That would imply that also the optical LINERs and Composite sources in the sample should be powered by SBN-dominated sources.

Acknowledgements. The authors are thankful for the discussions and contributions made by Aubrey Haschick (formerly at Haystack Observatory) and Elizabeth Besenfelder (formerly at Univ. North Carolina). The authors are thankful for the constructive comments made by the referee of this paper.

The Westerbork Observatory is part of the Netherlands Foundation for Research in Astronomy (NFRA-ASTRON) and is partially funded by the Organization for Scientific Research (NWO) of The Netherlands.

\section{References}

Araya, E., Baan, W. A., \& Hofner, P. 2004, ApJS, 154, 541

Baan, W. A. 1988, ApJ, 330, 743

Baan, W. A. 1989, ApJ, 338, 804

Baan, W. A. 1997, High Sensitivity Radio Astronomy, ed. N. Jackson, \& R. J. Davis, Cambridge, 73

Baan, W. A., \& Haschick, A. D. 1984, ApJ, 279, 541

Baan, W. A., \& Haschick, A. D. 1990, ApJ, 364, 65 (BH)

Baan, W. A., \& Haschick, A. D. 1995, ApJ, 454, 745
Baan, W. A., Salzer, J., \& LeWinter, R. 1998, ApJ, 509, 633

Baan, W. A., Haschick, A. D., \& Uglesich, R. 1993, ApJ, 415, 140

Condon, J. J. 1983, ApJS, 53, 459

Condon, J. J. 1992, ARA\&A, 575

Condon, J. J., \& Broderick, J. J. 1988, AJ, 96, 30

Condon, J. J., \& Broderick, J. J. 1991, AJ, 102, 1663

Condon, J. J., Helou, G., Sanders, D. B., \& Soifer, T. B. 1990, ApJS, 73, 359 (C90)

Condon, J. J., Frayer, D. T., \& Broderick, J. J. 1991a, AJ, 101, 362 (C91)

Condon, J. J., Huang, Z.-P., Yin, Q. F., \& Thuan, T. X. 1991b, ApJ, 378, 65 (CHYT)

Crawford, T., Marr, J., Partridge, B., \& Strauss, M. 1996, ApJ, 460, 225

Diamond, P. J., Norris, R. P., Baan, W. A., \& Booth, R. S. 1989, ApJ, 340, L49

Genzel, R., Lutz, D., Sturn, E., et al. 1998, ApJ, 498, 579

Gioia, I. M., Gregorini, L., \& Klein, U. 1982, A\&A, 116, 164

de Grijp, M. H. K., Miley, G. K., Lub, J., \& de Jong, T. 1985, Nature, 314,240

Heckman, T. 1998, The Most Distant Radio Galaxies, ed. Röttgering, P. Best, \& M. Lehnert (Dordrecht: D. Reidel), 227

Heckman, T., Armus, L., \& Miley, G. K. 1990, ApJS, 74, 833

Helou, G., Soifer, B. T., \& Rowan-Robinson, M. 1985, ApJ, 298, L7

Henkel, C., Baan, W. A., \& Mauersberger, R. 1992, A\&AR, 3, 47

Henkel, C., \& Wilson, T. 1990, A\&A, 229, 431

Klöckner, H.-R. 2004, Ph.D. Thesis, University of Groningen

Knapen, J. H., Laine, S., Yates, J. A., et al. 1997, ApJ, 490, 29

Lonsdale, C. J., Smith, H. E., \& Lonsdale, C. J. 1993, ApJ, 405, L9

Lutz, D., et al. 1996, A\&A, 315, L137

Mazzarella, J. M., Bothun, G., \& Boroson, T. 1991, AJ, 101, 2034

Majewski, S. R., Hereld, M., Koo, D. C., Illingworth, G. D., \& Heckman, T. M. 1993, ApJ, 402, 125

Martin, J. M. 1989, Thesis, Université de Paris

Miley, G. K., Neugebauer, G., \& Soifer, B. T. 1985, ApJ, 293, L11

Norris, R. P., Kesteven, M., Troup, E., Allen, D., \& Sramek, R. 1990, ApJ, 359, 291

Price, R., \& Duric, N. 1992, ApJ, 401, 81

Sanders, D. B., Soifer, B. T., Elias, J. H., et al. 1988, ApJ, 325, 74

Scoville, N. Z., Yun, M. S., \& Bryant, P. M. 1997, ApJ, 484, 702

Smith, H. E., Lonsdale, C. J., Lonsdale, C. J., \& Diamond, P. J. 1998, ApJ, 492, 137

Roy, A. L., Norris, R. P., Kesteven, M. J., Troup, E. R., \& Reynolds, J. E. 1994, Ap\&SS, 219, 67

Veilleux, S., Kim, D.-C., \& Sanders, D. B. 1999, ApJ, 522, 113

Veilleux, S., Kim, D.-C., Sanders, D. B. Mazzarella, J. M., \& Soifer, B. T. 1995, ApJS, 98, 171

Ward, M. J., Elvis, M., Fabbiano, G., et al. 1987, ApJ, 315, 74

Wilson, A. S. 1988, A\&A, 206, 41

Wunderlich, E., Wielebinski, R., \& Klein, U. 1987, A\&AS, 69, 487

Yun, M. S., Reddy, N. A., \& Condon, J. J. 2001, ApJ, 554, 803 
W. A. Baan and H.-R. Klöckner: Radio properties of FIR-megamaser nuclei, Online Material p 1

\section{Online Material}


W. A. Baan and H.-R. Klöckner: Radio properties of FIR-megamaser nuclei, Online Material p 2

\section{Appendix A: Radio maps of sample sources}
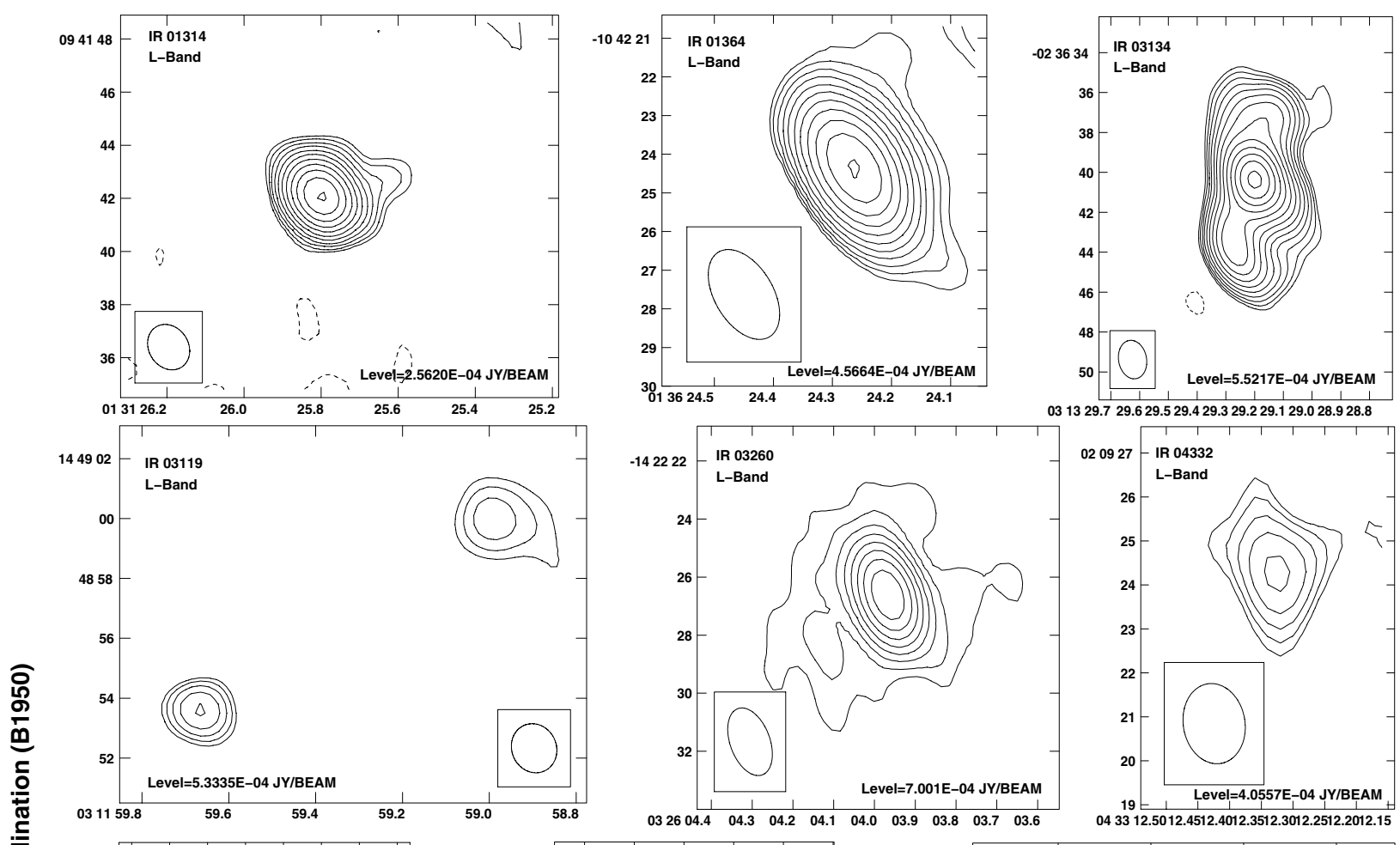

๑ั

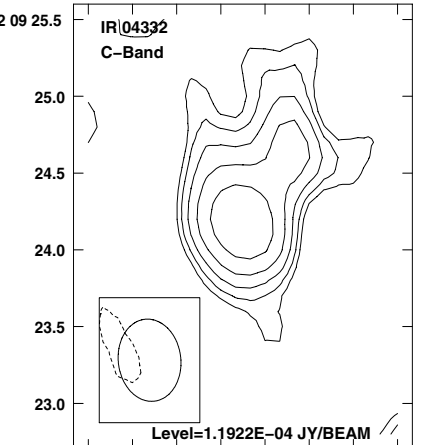

043312.4012 .3812 .3612 .3412 .3212 .3012 .2812 .26
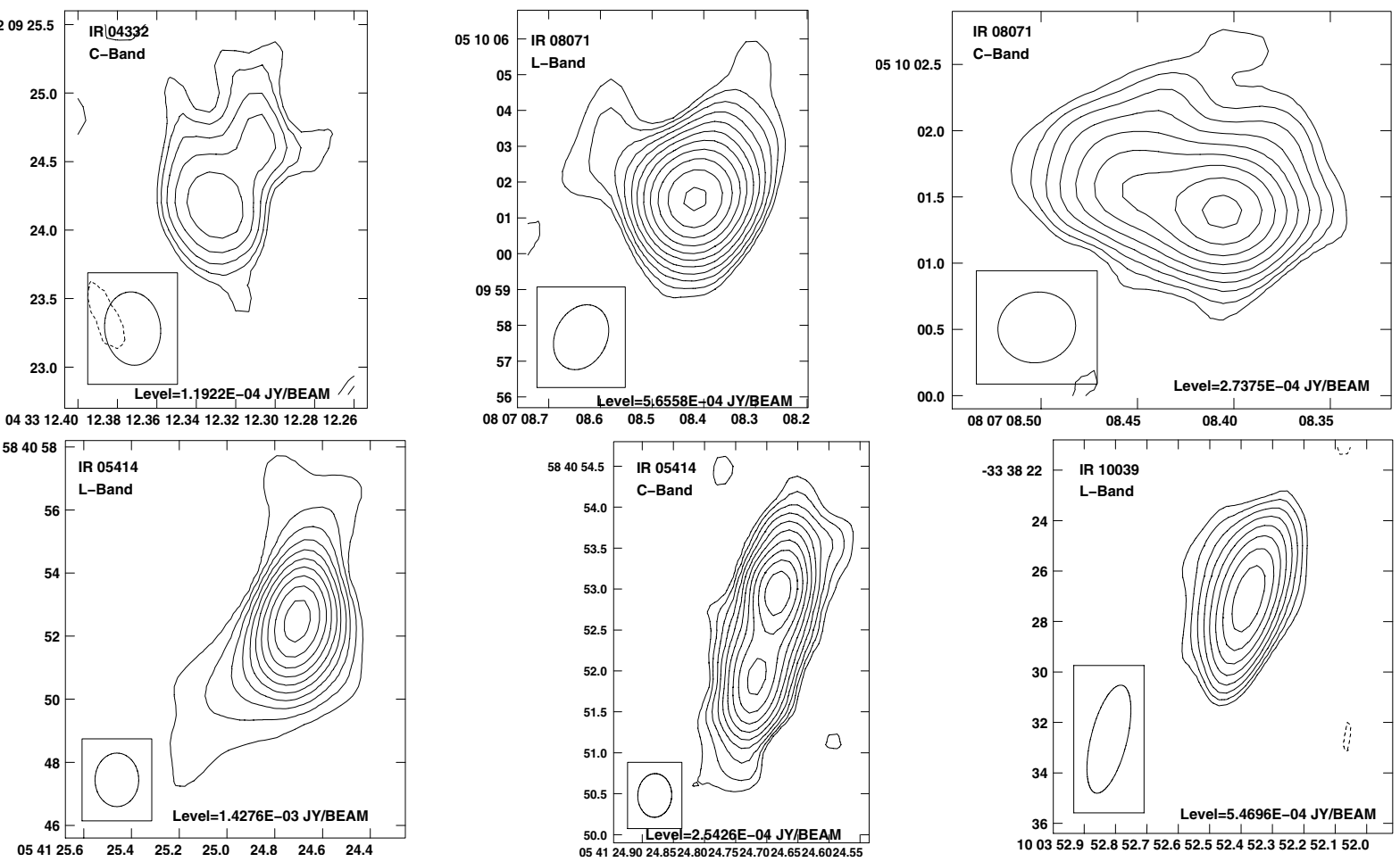

Right Ascension (B1950)

Fig. A.1. The radio maps of sample sources with extended structures. The levels given at the bottom of each frame designates the first contour in the map. The contours are at Level $\times \sqrt{2}^{n}(\mathrm{mJy} / \mathrm{beam})$. 
W. A. Baan and H.-R. Klöckner: Radio properties of FIR-megamaser nuclei, Online Material p 3
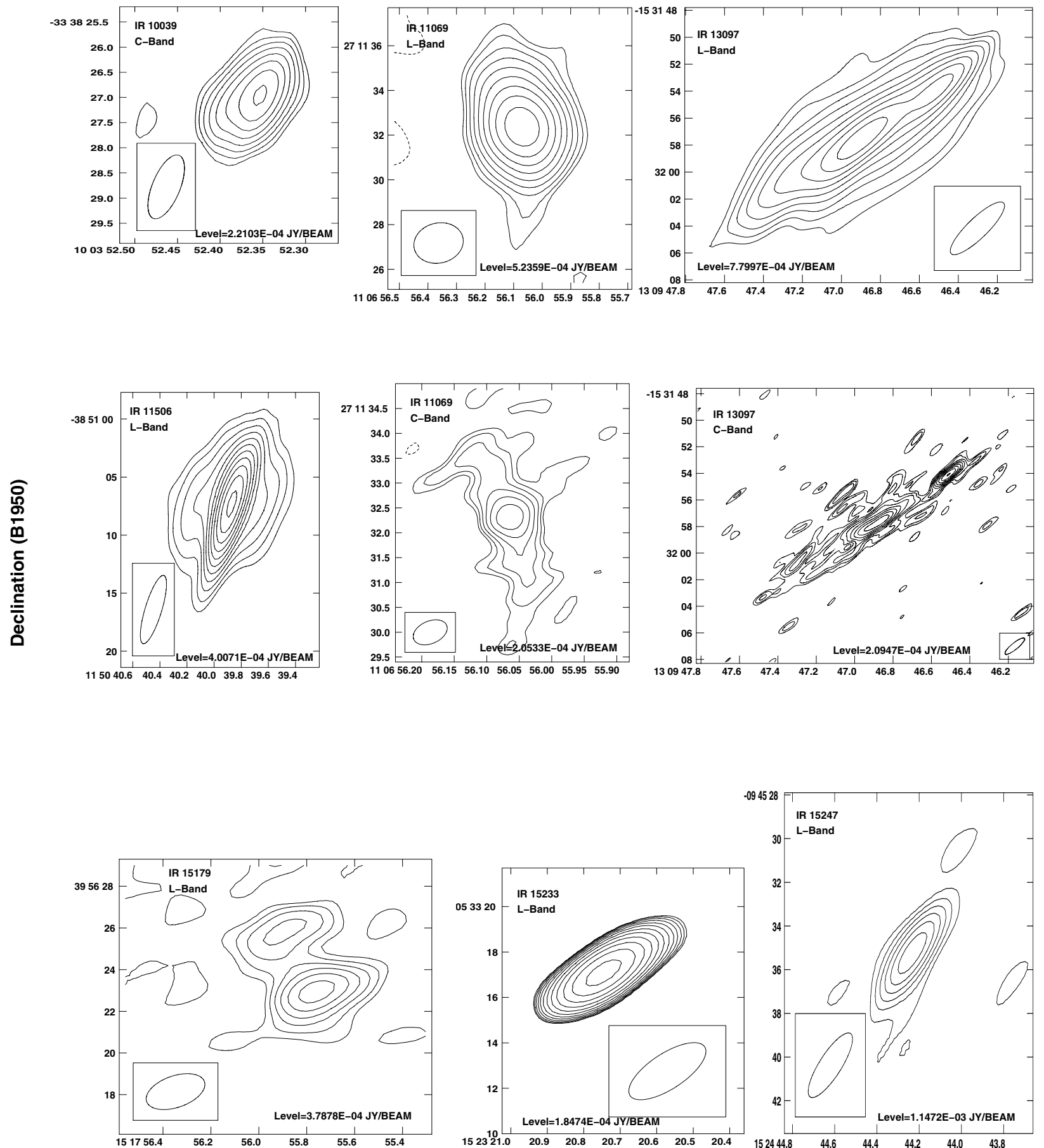

Right Ascension (B1950)

Fig. A.1. continued. 
W. A. Baan and H.-R. Klöckner: Radio properties of FIR-megamaser nuclei, Online Material p 4
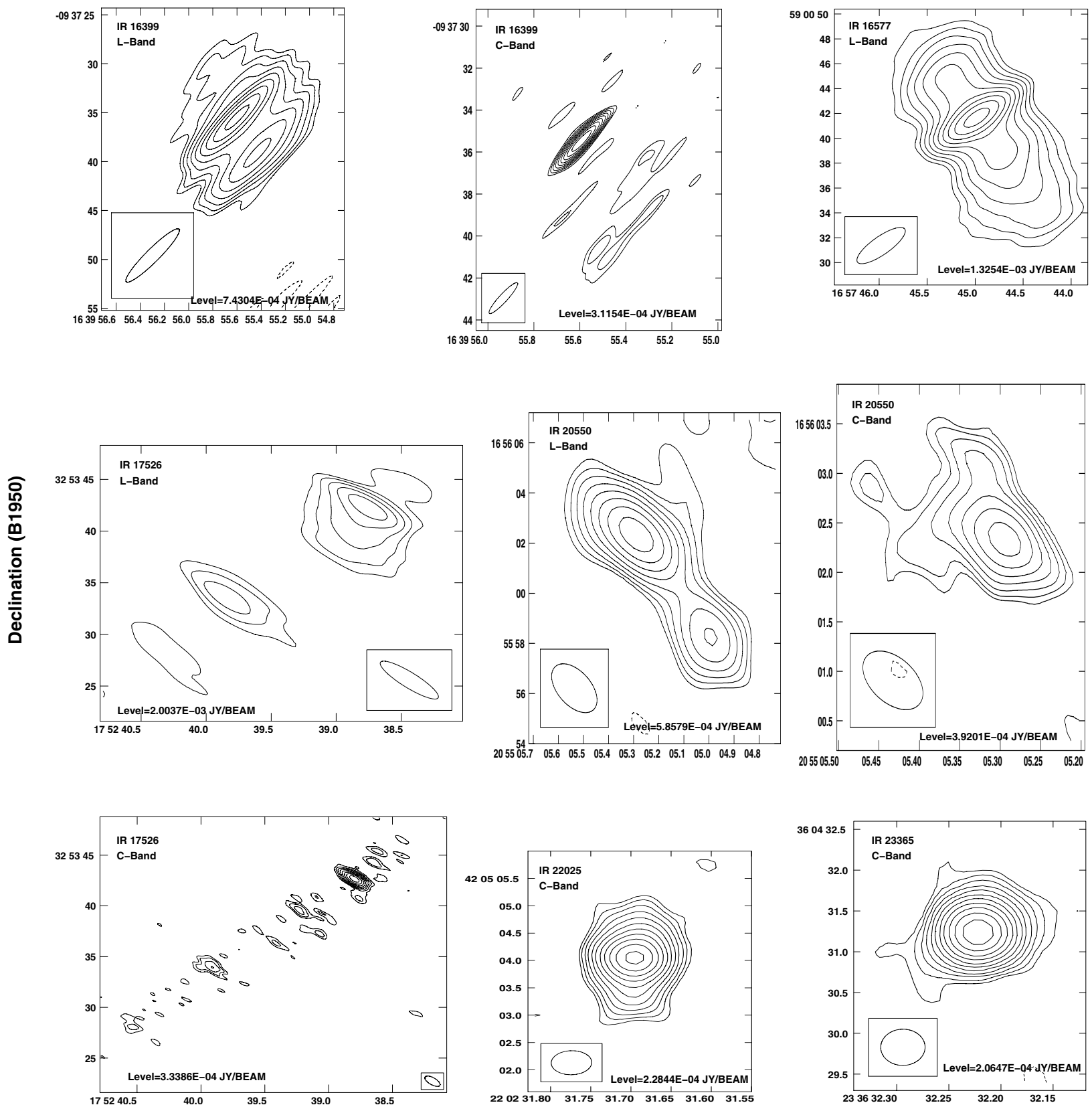

Right Ascension (B1950)

Fig. A.1. continued. 
W. A. Baan and H.-R. Klöckner: Radio properties of FIR-megamaser nuclei, Online Material p 5

\section{Appendix B: Tables}

Table B.1. Observational data.

\begin{tabular}{|c|c|c|c|c|c|c|c|c|c|c|c|c|c|}
\hline Name & & RA & Dec & (4) & $\begin{array}{l}\text { Freq. } \\
(\mathrm{GHz}) \\
(5)\end{array}$ & $\begin{array}{c}\text { Peak } \\
\text { Flux } \\
(\mathrm{mJy} / \mathrm{b}) \\
(6)\end{array}$ & $\begin{array}{c}\text { Total } \\
\text { Intensity } \\
(\mathrm{mJy}) \\
(7)\end{array}$ & $\begin{array}{c}\text { Size } \\
\theta_{M} \times \theta_{m} \\
(") \times(") \\
(8)\end{array}$ & $\begin{array}{l}\left({ }^{o}\right) \\
(9)\end{array}$ & $\begin{array}{c}(\mathrm{mJy} / \mathrm{b}) \\
(10)\end{array}$ & $\begin{array}{l}F_{60} \\
(\mathrm{Jy}) \\
(11)\end{array}$ & $\begin{array}{l}\text { (Jy) } \\
(12)\end{array}$ & $\begin{array}{l}\text { Comments } \\
\text { References } \\
\text { (13) }\end{array}$ \\
\hline \multirow[t]{2}{*}{ IR00057+4021 } & & 000545.14 & +402114.1 & 0.045 & 1.4 & 6.70 & 7.40 & $0.53 \times 0.45$ & 34 & 0.15 & 4.5 & 4.3 & MM \\
\hline & & & & & 4.8 & 4.07 & 4.20 & $0.08 \times 0.02$ & 34 & 0.09 & & & \\
\hline \multirow[t]{2}{*}{ IR00335+2732 } & & 003331.84 & -273204.6 & 0.069 & 1.4 & 13.3 & 14.7 & $0.54 \times 0.50$ & 61 & 0.31 & 4.3 & 3.2 & \\
\hline & & & & & 4.8 & 5.89 & 7.10 & $0.28 \times 0.20$ & 14 & 0.11 & & & \\
\hline \multirow[t]{2}{*}{ IR01314+0941 } & & 013125.80 & +094142.1 & 0.049 & 1.4 & 7.59 & 9.97 & $0.99 \times 0.66$ & 53 & 0.15 & 2.0 & 3.1 & \\
\hline & & & & & 4.8 & 2.66 & 3.24 & $0.27 \times 0.21$ & 157 & 0.08 & & & \\
\hline \multirow[t]{3}{*}{ IR01364+1042 } & & 013624.26 & -104224.3 & 0.047 & 1.4 & 15.0 & 15.3 & $0.29 \times 0.20$ & 87 & 0.23 & 6.2 & 6.7 & MM \\
\hline & & & & & 4.8 & 10.4 & 11.8 & $0.16 \times 0.16$ & - & 0.03 & & & \\
\hline & & & & & 8.4 & 6.40 & 8.00 & $0.19 \times 0.08$ & - & & & & CHYT \\
\hline IR01418+1651 & & 014147.91 & +165106.3 & 0.028 & 1.4 & 37.1 & 39.2 & $0.40 \times 0.40$ & - & 0.15 & 12.6 & 13.3 & MM, C90 \\
\hline 3Zw035 & & & & & 8.4 & 13.7 & 19.3 & $0.18 \times 0.14$ & - & 0.03 & & & CHYT \\
\hline \multirow[t]{2}{*}{ IR02483+4302 } & & 024818.49 & +430257.0 & 0.052 & 1.4 & 859 & 952 & $0.46 \times 0.13$ & 109 & 6.53 & 4.0 & 6.9 & MMC \\
\hline & & & & & 4.8 & 1060 & 1181 & $0.12 \times 0.05$ & 101 & 6.20 & & & \\
\hline \multirow[t]{2}{*}{ IR03056+2034 } & & 030538.59 & +203453.8 & 0.027 & 1.4 & 8.31 & 9.55 & $0.57 \times 0.42$ & 139 & 0.16 & 4.4 & 5.9 & Lext \\
\hline & & & & & 4.8 & 2.43 & 2.61 & $0.29 \times 0.20$ & 65 & 0.08 & & & \\
\hline \multirow[t]{3}{*}{ IR03119+1448 } & $\mathrm{N}$ & 031158.98 & +144859.9 & & 1.4 & 1.46 & 3.45 & $1.89 \times 1.65$ & 14 & 0.23 & 2.1 & 2.6 & \\
\hline & $\mathrm{MC}$ & 031159.66 & +144853.6 & 0.076 & 1.4 & 2.94 & 3.18 & $0.83 \times 0.83$ & 34 & 0.23 & & & D11.7 \\
\hline & & & & & 4.8 & 1.50 & 1.74 & $0.22 \times 0.22$ & - & 0.08 & & & \\
\hline IR03134+0236 & & 031329.20 & -023640.4 & 0.007 & 1.4 & 36.9 & 83.5 & $2.64 \times 1.81$ & 179 & 0.43 & 12.8 & 17.1 & OHabs, Lin \\
\hline \multirow[t]{2}{*}{ IR03260+1422 } & & 032603.97 & -142226.6 & 0.043 & 1.4 & 9.9 & 14.9 & $1.55 \times 1.08$ & 34 & 0.21 & 2.9 & 4.8 & MM, Lext \\
\hline & & & & & 4.8 & 5.69 & 6.04 & $0.15 \times 0.15$ & - & 0.09 & & & \\
\hline \multirow[t]{3}{*}{ IR04332+0209 } & $\mathrm{C}$ & 043312.32 & +020924.2 & 0.012 & 1.4 & 1.33 & 1.76 & $1.05 \times 0.61$ & 161 & 0.12 & 3.4 & 6.8 & MM \\
\hline & $\mathrm{C}$ & 043312.33 & +020924.2 & & 4.8 & 0.64 & 1.53 & $0.68 \times 0.46$ & 167 & 0.07 & & & \\
\hline & NW & 043312.31 & +020924.5 & & 4.8 & 0.42 & 1.03 & $0.94 \times 0.30$ & 178 & 0.07 & & & \\
\hline IR05414+5840 & M & 054124.70 & +584052.4 & 0.016 & 1.4 & 32.7 & 61.3 & $1.94 \times 0.62$ & 162 & 0.42 & 14.5 & 29.3 & OHabs \\
\hline \multirow[t]{2}{*}{ UGC 03351} & $\mathrm{~N}$ & 054124.68 & +584052.9 & & 4.8 & 5.93 & 14.6 & $0.88 \times 0.26$ & 167 & 0.12 & & & Lin \\
\hline & $\mathrm{S}$ & 054124.71 & +584052.0 & & 4.8 & 3.53 & 11.2 & $1.07 \times 0.32$ & 165 & 0.12 & & & \\
\hline \multirow[t]{2}{*}{ IR06206+3646 } & & 062038.61 & -364611.3 & 0.108 & 1.4 & 19.8 & 20.8 & $0.54 \times 0.38$ & 8 & 0.30 & 2.2 & 2.5 & MM \\
\hline & & & & & 4.8 & 12.4 & 13.5 & $0.18 \times 0.13$ & 154 & 0.16 & & & \\
\hline \multirow[t]{3}{*}{ IR08071+0510 } & M & 080708.41 & +051001.4 & 0.052 & 1.4 & 17.7 & 28.2 & $1.27 \times 0.98$ & 70 & 0.26 & 5.0 & 6.9 & MM \\
\hline & $\mathrm{C}$ & 080708.41 & +051001.4 & & 4.8 & 4.62 & 8.65 & $0.63 \times 0.43$ & 78 & 0.08 & & & Cext \\
\hline & $\mathrm{E}$ & 080708.43 & +051001.5 & & 4.8 & 2.75 & 9.77 & $1.31 \times 0.57$ & 70 & 0.08 & & & Cext \\
\hline IR09320+6134 & & 093204.78 & +613437.0 & 0.040 & 1.4 & 116 & 132 & $0.80 \times 0.50$ & - & 0.15 & 11.5 & 20.2 & MM, C90 \\
\hline UGC 05101 & & & & & 8.4 & 32.6 & 41.8 & $0.14 \times 0.11$ & - & 0.03 & & & CHYT \\
\hline \multirow[t]{2}{*}{ IR10039-3338 } & & 100352.37 & -333827.1 & 0.034 & 1.4 & 7.10 & 10.3 & $1.78 \times 1.01$ & 139 & 0.24 & 8.9 & 7.9 & MM \\
\hline & & & & & 4.8 & 2.66 & 2.85 & $0.42 \times 0.21$ & 19 & 0.12 & & & \\
\hline \multirow[t]{2}{*}{ IR10173+0828 } & & 101722.26 & +082839.7 & 0.048 & 1.4 & 8.20 & 8.40 & $0.40 \times 0.40$ & - & 0.15 & 5.8 & 5.5 & MM, C90 \\
\hline & & & & & 8.4 & 4.90 & 5.30 & $0.08 \times 0.05$ & - & 0.03 & & & CHYT \\
\hline \multirow[t]{2}{*}{ IR10378+1109 } & & 103750.92 & +110858.5 & 0.136 & 1.4 & 6.99 & 8.71 & $1.25 \times 0.27$ & 71 & 0.83 & 2.3 & 1.8 & MM \\
\hline & & & & & 4.8 & 4.17 & 4.39 & $0.14 \times 0.10$ & 104 & 0.10 & & & \\
\hline \multirow[t]{2}{*}{ IR10485-1447 } & & 104834.69 & -144726.2 & 0.133 & 1.4 & 5.74 & 6.45 & $0.81 \times 0.26$ & 2 & 0.23 & 1.7 & 1.7 & MMC \\
\hline & & & & & 4.8 & 3.13 & 3.37 & $0.17 \times 0.11$ & 100 & 0.10 & & & \\
\hline IR11010+4107 & & 110105.81 & +410710.5 & 0.035 & 1.4 & 16.6 & 24.3 & $1.30 \times 0.70$ & - & 0.15 & 5.8 & 10.6 & MM, C90 \\
\hline
\end{tabular}


W. A. Baan and H.-R. Klöckner: Radio properties of FIR-megamaser nuclei, Online Material p 6

Table B.1. continued.

\begin{tabular}{|c|c|c|c|c|c|c|c|c|c|c|c|c|c|}
\hline Name & & RA & Dec & $(4)$ & Freq. & $\begin{array}{c}\text { Peak } \\
\text { Flux } \\
(\mathrm{mJy} / \mathrm{b}) \\
(6)\end{array}$ & $\begin{array}{c}\text { Total } \\
\text { Intensity } \\
(\mathrm{mJy}) \\
(7)\end{array}$ & $\begin{array}{c}\text { Size } \\
\theta_{M} \times \theta_{m} \\
(") \times(") \\
(8)\end{array}$ & $\begin{array}{l}\mathrm{PA} \\
\left({ }^{o}\right) \\
(9)\end{array}$ & $\begin{array}{c}\mathrm{rms} \\
(\mathrm{mJy} / \mathrm{b}) \\
(10)\end{array}$ & $\begin{array}{l}F_{60} \\
(\mathrm{Jy}) \\
(11)\end{array}$ & $\begin{array}{l}F_{100} \\
(\mathrm{Jy}) \\
(12)\end{array}$ & $\begin{array}{l}\text { Comments } \\
\text { References } \\
\text { (13) }\end{array}$ \\
\hline A1101+41 & & & & & 8.4 & 5.60 & 8.00 & $0.22 \times 0.10$ & - & 0.03 & & & CHYT \\
\hline \multirow[t]{4}{*}{ IR11069+2711 } & M & I 110656.06 & +271132.4 & 0.070 & 1.4 & 9.01 & 16.0 & $2.27 \times 0.62$ & 22 & 0.22 & 2. & 4.1 & Lext \\
\hline & $\mathrm{C}$ & 110656.06 & +271132.3 & & 4.8 & 1.47 & 3.87 & $0.92 \times 0.43$ & 27 & 0.08 & & & Cext, Lin \\
\hline & $\mathrm{NE}$ & 110656.09 & +271133.3 & & 4.8 & 0.53 & 3.35 & $1.76 \times 0.89$ & 34 & 0.08 & & & \\
\hline & SW & 110656.06 & +271132.0 & & 4.8 & 0.58 & 3.50 & $1.7 \times 0.84$ & 20 & 0.08 & & & \\
\hline IR11257+5850 & $\mathrm{M}$ & I 112544.19 & +585018.3 & 0.010 & 1.4 & 132. & 658. & $0.70 \times 0.90$ & & 0.15 & 103.7 & 107.4 & MM, Lext \\
\hline N3690, IC 694 & & & & & 8.4 & 29.0 & 78.3 & $0.38 \times 0.28$ & - & 0.03 & & & C90, CHYT, BH \\
\hline \multirow[t]{2}{*}{ IR11506-3851 } & & 115039.86 & -385107.3 & 0.009 & 1.4 & 54.5 & 64.4 & $2.21 \times 0.57$ & 167 & 0.78 & 32.7 & 41.6 & MM \\
\hline & & & & & 4.8 & 24.5 & 32.1 & $0.36 \times 0.27$ & 168 & 0.32 & & & \\
\hline \multirow[t]{2}{*}{ IR12018+1941 } & & 120151.11 & +194151.7 & 0.169 & 1.4 & 5.45 & 6.12 & $1.19 \times 0.35$ & 98 & 0.21 & 1.8 & 1.8 & MM \\
\hline & & & & & 4.8 & 2.54 & 2.82 & $0.33 \times 0.10$ & 97 & 0.09 & & & \\
\hline \multirow[t]{4}{*}{ IR12112+0305 } & $\mathrm{MN}$ & 121112.48 & +030522.1 & 0.073 & 1.4 & 16.2 & 18.5 & $0.60 \times 0.50$ & - & 0.15 & 8.5 & 9.9 & MM, C90 \\
\hline & & & & & 8.4 & 6.30 & 7.60 & $0.12 \times 0.09$ & - & 0.03 & & & CHYT \\
\hline & MS & 121112.37 & +030519.7 & 0.073 & 1.4 & 4.20 & 4.20 & $0.50 \times 0.50$ & - & 0.15 & & & $\mathrm{C} 90$ \\
\hline & & & & & 8.4 & 1.30 & 1.70 & $0.19 \times 0.13$ & - & 0.03 & & & CHYT \\
\hline IR12243-0036 & & 122420.75 & -003603.6 & 0.007 & 1.4 & 36.0 & 38.5 & $0.50 \times 0.30$ & - & 0.15 & 40.7 & 32.8 & MM, C90 \\
\hline NGC 4418 & & & & & 4.8 & 23.8 & 26.1 & $0.18 \times 0.02$ & 44 & 0.29 & & & \\
\hline IR12540+5708 & & 125405.01 & +570838.2 & 0.042 & 1.4 & 227. & 237. & $0.40 \times 0.30$ & - & 0.15 & 31.9 & 30.3 & MM, C90 \\
\hline UGC 08058 & & & & & 8.4 & 248. & 261. & $0.07 \times 0.06$ & - & 0.03 & & & CHYT \\
\hline \multirow[t]{6}{*}{ IR13097-1531 } & NW & 130946.51 & -153154.1 & 0.022 & 1.4 & 8.94 & 13.8 & $2.73 \times 1.45$ & 132 & 0.36 & 11.1 & 20.9 & MMC, D6.6, Lin \\
\hline & NW & 130946.51 & -153154.1 & & 4.8 & 3.22 & 3.96 & $0.71 \times 0.27$ & 131 & 0.09 & & & \\
\hline & & 130946.89 & -153157.6 & 0.022 & 1.4 & 13.0 & 31.6 & $5.38 \times 1.73$ & 122 & 0.36 & & & \\
\hline & & 130946.88 & -153157.8 & & 4.8 & 2.71 & 10.4 & $3.12 \times 0.78$ & 120 & 0.08 & & & Cext, Multi \\
\hline & SE1 & 130947.26 & -153201.0 & & 4.8 & 0.48 & 6.86 & $4.49 \times 2.85$ & 118 & 0.08 & & & \\
\hline & SE2 & 130947.08 & -153200.1 & & 4.8 & 0.54 & 6.98 & $7.06 \times 1.51$ & 120 & 0.08 & & & \\
\hline IR13126+2452 & & 131239.49 & +245258.1 & 0.013 & 1.4 & 29.4 & 31.3 & $0.40 \times 0.30$ & - & 0.15 & 17.9 & 18.1 & MM, Form, C90 \\
\hline IC 860 & & & & & 4.8 & 19.0 & 21.0 & $0.21 \times 0.17$ & 104 & 0.21 & & & OHabs+em \\
\hline \multirow{2}{*}{ IR13254+4754 } & & 132526.07 & +475434.8 & 0.059 & 1.4 & 7.20 & 8.44 & $0.75 \times 0.61$ & 138 & 0.18 & 1.9 & 2.7 & MMC \\
\hline & & & & & 4.8 & 2.71 & 3.06 & $0.24 \times 0.12$ & 82 & 0.10 & & & \\
\hline IR13428+5608 & & 134251.71 & +5608 14.3 & 0.038 & 1.4 & 80.3 & 91.2 & $0.80 \times 0.80$ & - & 0.15 & 21.7 & 21.4 & MM, D0.8, disk \\
\hline UGC 08696 & & & & & 8.4 & 20.3 & 34.8 & $0.32 \times 0.18$ & - & 0.03 & & & C90, СHYT \\
\hline \multirow[t]{2}{*}{ Mrk 273} & & 134251.76 & +560813.6 & 0.038 & 1.4 & 9.80 & 38.2 & $3.50 \times 1.70$ & - & 0.15 & 0.0 & 0.0 & C90 \\
\hline & & & & & 8.4 & 3.20 & 3.90 & $0.18 \times 0.11$ & - & 0.03 & & & CHYT \\
\hline \multirow[t]{2}{*}{ IR13451+1232 } & & 134506.17 & +123220.3 & 0.121 & 1.4 & 5109 & 5155 & $0.25 \times 0.25$ & - & 56.6 & 1.9 & 2.1 & \\
\hline & & & & & 4.8 & 2832 & 2931 & $0.14 \times 0.05$ & 136 & 28.8 & & & \\
\hline \multirow[t]{2}{*}{ IR14070+0525 } & & 140700.77 & +052540.7 & 0.265 & 1.4 & 5.33 & 5.49 & $0.75 \times 0.75$ & - & 0.18 & 1.5 & 1.8 & MM \\
\hline & & & & & 4.8 & 2.67 & 2.75 & $0.34 \times 0.34$ & - & 0.09 & & & \\
\hline IR14566-1629 & & 145637.03 & -162941.5 & 0.012 & 1.4 & 983 & 1047 & $0.54 \times 0.12$ & 103 & 10.4 & 6.2 & 8.9 & $\mathrm{OH}$ Abs \\
\hline NGC 5793 & & & & & 4.8 & 444 & 508 & $0.29 \times 0.10$ & 117 & 4.27 & & & \\
\hline IR15107+0724 & & 151045.69 & +072442.4 & 0.013 & 1.4 & 45.7 & 51.0 & $0.50 \times 0.50$ & - & 0.15 & 20.8 & 29.4 & MM, Form, C90 \\
\hline Zw049.057 & & & & & 4.8 & 29.3 & 33.2 & $0.25 \times 0.19$ & 115 & 0.30 & & & \\
\hline \multirow[t]{3}{*}{ IR15179+3956 } & $\mathrm{C}$ & 151755.75 & +3956 22.9 & 0.047 & 1.4 & 2.31 & 4.55 & $2.30 \times 1.79$ & 98 & 0.17 & 4.2 & 3.1 & MM, D3.5 \\
\hline & $\mathrm{C}$ & & & & 4.8 & 1.25 & 1.53 & $0.33 \times 0.07$ & 144 & 0.10 & & & \\
\hline & $\mathrm{NE}$ & 151755.89 & +395625.5 & & 1.4 & 1.08 & 3.85 & $3.98 \times 2.83$ & 105 & 0.17 & & & \\
\hline \multirow[t]{2}{*}{ IR15233+0533 } & & 152320.75 & +053317.2 & 0.055 & 1.4 & 12.1 & 13.7 & $1.09 \times 1.09$ & - & 0.40 & 3.5 & 3.9 & \\
\hline & & & & & 4.8 & 4.80 & 5.23 & $0.23 \times 0.23$ & - & 0.12 & & & \\
\hline \multirow[t]{2}{*}{ IR15247-0945 } & & 152444.23 & -094535.3 & 0.040 & 1.4 & 7.08 & 12.8 & $3.08 \times 1.36$ & 134 & 0.36 & 4.7 & 6.0 & MM \\
\hline & & & & & 4.8 & 4.35 & 4.82 & $0.46 \times 0.11$ & 133 & 0.11 & & & \\
\hline
\end{tabular}


W. A. Baan and H.-R. Klöckner: Radio properties of FIR-megamaser nuclei, Online Material p 7

Table B.1. continued.

\begin{tabular}{|c|c|c|c|c|c|c|c|c|c|c|c|c|}
\hline Name & $\overline{\mathrm{RA}}$ & Dec & (4) & Freq. & $\begin{array}{c}\text { Peak } \\
\text { Flux } \\
(\mathrm{mJy} / \mathrm{b}) \\
(6)\end{array}$ & $\begin{array}{c}\text { Total } \\
\text { Intensity } \\
(\mathrm{mJy}) \\
(7)\end{array}$ & $\begin{array}{c}\text { Size } \\
\theta_{M} \times \theta_{m} \\
(") \times(") \\
(8)\end{array}$ & $\begin{array}{l}\left({ }^{o}\right) \\
(9)\end{array}$ & $\begin{array}{c}\mathrm{rms} \\
(\mathrm{mJy} / \mathrm{b}) \\
(10)\end{array}$ & $\begin{array}{l}F_{60} \\
(\mathrm{Jy}) \\
(11)\end{array}$ & $\begin{array}{l}F_{100} \\
(\mathrm{Jy}) \\
(12)\end{array}$ & $\begin{array}{l}\text { Comments } \\
\text { References } \\
\text { (13) }\end{array}$ \\
\hline \multirow[t]{2}{*}{ IR15250+3609 } & 152503.72 & +360901.0 & 0.055 & 1.4 & 12.5 & 12.8 & $0.30 \times 0.30$ & - & 0.15 & 7.3 & 5.9 & MM, C90 \\
\hline & & & & 8.4 & 9.70 & 10.2 & $0.06 \times 0.05$ & - & 0.03 & & & CHYT \\
\hline IR15327+2340 & C 153246.92 & +234007.8 & 0.018 & 1.4 & 205. & 299. & $1.30 \times 0.60$ & - & 0.15 & 103.8 & 112.4 & MM, Form, D0.93 \\
\hline UGC 09913 & E 153246.88 & +234007.9 & & 8.4 & 46.8 & 72.2 & $0.21 \times 0.14$ & - & 0.03 & & & C90, CHYT \\
\hline ARP 220 & W 153246.95 & +234007.7 & & 8.4 & 28.5 & 60.7 & $0.32 \times 0.19$ & - & 0.03 & & & \\
\hline \multirow[t]{3}{*}{ IR16399-0937 } & C 163955.60 & -093735.6 & 0.026 & 1.4 & 12.0 & 18.3 & $3.28 \times 1.48$ & 136 & 0.37 & 8.0 & 12.5 & MM, Lin \\
\hline & C 163955.59 & -093735.5 & & 4.8 & 6.75 & 7.61 & $0.47 \times 0.18$ & 116 & 0.15 & & & Cext, Multi \\
\hline & SW 163955.45 & -093739.0 & & 1.4 & 12.0 & 21.9 & $5.37 \times 1.52$ & 133 & 0.37 & & & \\
\hline IR16577+5900 & C 165744.98 & +590041.7 & 0.019 & 1.4 & 30.9 & 64.7 & $2.46 \times 1.77$ & 44 & 0.47 & 7.9 & 22.6 & OHabs, Lin \\
\hline \multirow[t]{3}{*}{ NGC $6285 / 6$} & $\mathrm{C}$ & & & 8.4 & 5.80 & - & $0.25 \times 0.25$ & - & 0.03 & & & C90, CHYT \\
\hline & NE 165745.22 & +590044.1 & & 1.4 & 8.82 & 46.0 & $5.83 \times 3.66$ & 24 & 0.46 & & & \\
\hline & SW 165745.27 & +590045.5 & & 1.4 & 13.8 & 23.8 & $18.6 \times 4.22$ & 31 & 0.34 & & & \\
\hline \multirow[t]{2}{*}{ IR17208-0014 } & 172047.86 & -001415.8 & 0.018 & 1.4 & 88.0 & 96 & $0.87 \times 0.87$ & - & 1.21 & 31. & 34.9 & MM \\
\hline & & & & 4.8 & 41.9 & 53.8 & $0.46 \times 0.26$ & 96. & 0.57 & & & \\
\hline IR17526+3253 & N 175238.79 & +325341.3 & 0.025 & 1.4 & 7.06 & 43.3 & $7.13 \times 5.24$ & 132 & 0.80 & 3.2 & 7.4 & MMC, Lext, Lin \\
\hline \multirow[t]{6}{*}{ UGC 11035} & N 175238.80 & +325342.7 & & 4.8 & 5.16 & 6.41 & $0.89 \times 0.32$ & 60 & 0.12 & & & Cext, Multi \\
\hline & SE1 175239.22 & +32 5339.6 & & 4.8 & 0.49 & 4.17 & $3.45 \times 2.47$ & 82 & 0.11 & & & \\
\hline & SE2 175239.80 & +325333.5 & & 1.4 & 4.53 & 19.9 & $1.96 \times 1.03$ & 86 & 0.80 & & & \\
\hline & SE2 175239.93 & +325334.0 & & 4.8 & 0.70 & 4.00 & $2.72 \times 1.82$ & 85 & 0.11 & & & \\
\hline & SE3 175240.29 & +325328.9 & & 1.4 & 2.26 & 2.42 & $16.4 \times 4.58$ & 42 & 0.80 & & & \\
\hline & SE3 175240.54 & +325328.3 & & 4.8 & 0.46 & 2.60 & $2.32 \times 2.18$ & 109 & 0.11 & & & \\
\hline \multirow[t]{2}{*}{ IR20491+1846 } & 204908.06 & +184645.9 & 0.029 & 1.4 & 1.88 & 4.48 & $4.39 \times 1.11$ & 18 & 0.42 & 2.8 & 6.2 & \\
\hline & & & & 4.8 & 0.30 & 0.71 & $2.00 \times 0.50$ & - & 0.13 & & & \\
\hline IR20550+1656 & C 205505.29 & +165602.3 & 0.037 & 1.4 & 7.73 & 16.1 & $3.36 \times 1.39$ & 64 & 0.47 & 13.3 & 10.6 & MM, D5.8 \\
\hline \multirow[t]{2}{*}{ 2ZW96 } & C 205505.30 & +165602.4 & & 4.8 & 3.21 & 6.57 & $1.07 \times 0.54$ & 63 & 0.22 & & & Cext \\
\hline & SW 205505.00 & +165558.4 & & 1.4 & 3.34 & 7.25 & $2.74 \times 1.80$ & 40 & 0.47 & & & \\
\hline \multirow[t]{2}{*}{ IR22025+4205 } & 220231.69 & +420504.0 & 0.016 & 1.4 & 21.5 & 22.4 & $0.57 \times 0.57$ & - & 0.74 & 9.7 & 14.0 & MM \\
\hline & & & & 4.8 & 7.24 & 12.8 & $0.48 \times 0.31$ & 169 & 0.11 & & & \\
\hline \multirow[t]{2}{*}{ IR22088-1831 } & 220849.85 & -183155.5 & 0.170 & 1.4 & 6.83 & 9.29 & $1.83 \times 1.83$ & 56 & 0.26 & 1.7 & 1.7 & MM \\
\hline & & & & 4.8 & 9.49 & 9.76 & $0.17 \times 0.17$ & - & 0.12 & & & \\
\hline \multirow[t]{2}{*}{ IR22491-1808 } & 224909.09 & -180820.6 & 0.076 & 1.4 & 4.30 & 5.80 & $1.40 \times 1.00$ & - & 0.15 & 5.4 & 4.5 & MM, C90 \\
\hline & & & & 8.4 & 2.60 & 2.80 & $0.09 \times 0.05$ & - & 0.03 & & & CHYT \\
\hline \multirow[t]{2}{*}{ IR23050+0359 } & 230503.12 & +035947.3 & 0.047 & 1.4 & 8.55 & 41.13 & $5.35 \times 2.61$ & 99 & 0.21 & 3.9 & 5.3 & \\
\hline & & & & 4.8 & 5.17 & 6.15 & $0.24 \times 0.18$ & 77 & 0.09 & & & \\
\hline IR23135+2517 & 231333.13 & +251701.6 & 0.027 & 1.4 & 13.1 & 24.2 & $1.40 \times 1.30$ & - & 0.15 & 9.7 & 11.1 & MM, C90 \\
\hline Zw475.056 & & & & 8.4 & 3.80 & 4.50 & $0.12 \times 0.09$ & - & 0.03 & & & CHYT, D2.0 \\
\hline \multirow[t]{2}{*}{ IR23365+3604 } & 233632.22 & +360431.2 & 0.064 & 1.4 & 20.9 & 23.5 & $0.53 \times 0.38$ & 125 & 0.32 & 7.1 & 8.4 & MM \\
\hline & & & & 4.8 & 7.78 & 10.2 & $0.26 \times 0.19$ & 110 & 0.11 & & & \\
\hline
\end{tabular}

\section{Footnote 1: The spectral and structural information}

The tabular information for the galaxies has the following order:

Column (1) - IRAS name and catalog galaxy name. The designations, M, N, S, W, and E, indicate the Main, North, South, West, and East components of the sources.

Columns (2) and (3) - Right ascension and declination.

Column (4) - Redshift.

Column (5) - Observing frequency in GHz.

Columns (6) through (10) - The peak flux (mJy/beam), the integrated flux (mJy), the deconvolved FWHM major diameter $\theta_{M}$ and minor diameter $\theta_{m}$ of the source in arcseconds, the position angle of the emission structure, and the rms noise (mJy/beam) of the map for each observation. 
Columns (11) and (12) - IRAS $60 \mu \mathrm{m}$ and $100 \mu \mathrm{m}$ fluxes in Jy.

Column (13) - Comments and references to observational data. The designation U stands for "unresolved", "Lext" and "Cext" signifies extended at $L$-band or at $C$-band, and "Dx" signifies a "double source with $\mathrm{x}$ separation in arcseconds". "Lin" indicates that a linear structure can be seen in the galaxy, resembling the emission of a central disk system. "MM" designates the sources with confirmed and published OH emission, while "MMC" designates sources that require further confirmation of the OH emission (present authors; Martin 1989). "Form" designates the three sources that also exhibit $\mathrm{H}_{2} \mathrm{CO}$ emission (see Baan et al. 1993; Araya et al. 2004). "Multi" indicates that the emission breaks up in multiple components. References for continuum or line information are designated with $\mathrm{r}$.

\section{Footnote 2: Comments on extended sources and sources deserving special mention.}

The optical orientation (anti-clockwise from North) is always designated by PA(opt) and all other PA values relate to the radio data. Maps for most of these sources are presented in Fig. A.1.

IR01314+0941 $-L$-band extension at $\mathrm{PA}=-78^{\circ}$. Unresolved at $C$-band. $\mathrm{NC}=000$.

IR03119+1448 - Double system with $L$-band unresolved sources at PA(double) $=-57^{\circ}$ and with separation of $17.2 \mathrm{kpc}$. $C$-band southern component only. $\mathrm{NC}=000$.

IR03134-0236 - OH absorber. Extended disc/triple source at $\mathrm{PA}=13^{\circ}$. No $\mathrm{C}$ data available.

IR03260-1422 $-L$-band halo extension at $\mathrm{PA}=157^{\circ}$. No structure at $C$-band. NC $=000$.

IR04332+0209 $-C$-band extended disk (double) at $\mathrm{PA}($ disk $)=-30^{\circ} . \mathrm{NC}=000$.

IR05414+5840 - UGC 03351 has nuclear $C$-band double/disk structure at PA(double $)=-14^{\circ}$. Symmetric $L$-band extensions. NC $=000$.

IR08071+0510 - Nuclear source with $L$-band extension at $\mathrm{PA}=74^{\circ} . C$-band extension at $\mathrm{PA}=88^{\circ} . \mathrm{NC}=000$.

IR09320+6134 - Nuclear source within extended disk (C90; C91). NC $=110$.

IR10039-3338 - L-band extension at $\mathrm{PA}=-20^{\circ} . \mathrm{NC}=000$.

IR11010+4107 - Nuclear source within extended disk (C90; C91). $\mathrm{NC}=000$.

$I R 11069+2711-L$-band extended at $\mathrm{PA}=10^{\circ}$ and nuclear $C$-band extended triple at $\mathrm{PA}=20^{\circ} . \mathrm{NC}=000$.

IR11257+5850 - IC 694 is part of an interaction system with NGC 3690 and also designated as Arp 299. Observational data is in the literature (C90; C91; Baan \& Haschick 1990). The megamaser occurs at the eastern nucleus of the system in IC 694 . IC 694 has a slight extension surrounding the compact nucleus with $\mathrm{PA}($ disk $)=\mathrm{PA}(\mathrm{opt})=-45^{\circ} . \mathrm{NC}=100$.

IR11506-3851 - Possibly weak $L$-band elongation at PA(ext $)=80^{\circ}$. Unresolved at $C$-band. $\mathrm{NC}=000$.

IR13079-1531 - Triple $L$-band disk structure at PA $=122^{\circ} . C$-band multiple component SNR-like structure with size $6.4 \mathrm{kpc} . \mathrm{NC}=000$.

IR13428+5608 - Mrk 273, UGC 08696 is a strongly interacting and merging binary (C90; C91; Knapen et al. 1997). The spatial separation of the two nuclei is $611 \mathrm{pc}$. Southern source lies below the apparent disk extension of northern source. NC $=000$.

IR13451+1232 - Radio loud nuclear source with no extension at $C$-band. NC $=110$.

IR14566-1629 - NGC 5793 is an OH absorber in radio-loud (dominant) nuclear source with slightly elongated structure. PA(ext) $=-35^{\circ}$. Possible $C$-band structure perpendicular to disk at $\mathrm{PA}=103^{\circ}$. $\mathrm{PA}$ (optical) $=-60^{\circ}$. NC $=110$.

IR15179+3956 - Interacting double with nuclear separation of $3.0 \mathrm{kpc}$ with weak third component off-axis. Only S-W component extended at $L$-band at $\mathrm{PA}=28^{\circ} . \mathrm{NC}=000$.

IR15247-0945 - Interacting double with nuclear separation of $12.5 \mathrm{kpc}$. North component not seen at $C$-band. NC $=001$.

IR15327+2340 - Arp 220 is the first detected $\mathrm{OH}$ megamaser, which also shows $\mathrm{H}_{2} \mathrm{CO}$ emission. Interacting nuclei with projected separation of 318 pc (see C90; C91; Baan et al. 1984; Diamond et al. 1989). Nuclear interaction described by Baan \& Haschick (1995) and Scoville et al. (1996). NC $=100$.

IR16399-0937 - Triple/multiple structure embedded within extended disk at $L$-band with PA(triple) $=27^{\circ}$. $C$-band structure composed of multiple SNR-like distribution at same PA as $L$-band structure. $\mathrm{NC}=000$.

IR16577+5900 - NGC 6285/6 is an $\mathrm{OH}$ absorber galaxy and part of interacting system. Triple nuclear source within extended disk with $\mathrm{PA}($ triple $)=33^{\circ} . \mathrm{PA}($ optical $)=38^{\circ}$. Unresolved central component remains at $8.4 \mathrm{GHz}(\mathrm{C} 91)$. $\mathrm{NC}=000$.

IR17526+3253 - UGC 11035 is a triple source with total extend of $16.8 \mathrm{kpc}$ at $L$-band at $\mathrm{PA}=119^{\circ}$. Central components seen at $C$-band. $\mathrm{PA}($ optical $)=135^{\circ} . \mathrm{NC}=000(\mathrm{~S})$ and $001(\mathrm{~N})$.

IR20550+1656 - 2Zw96 is a double system at $L$-band at PA $=37^{\circ}$ with projected nuclear separation of $4.3 \mathrm{kpc}$. Southern source not seen at $C$-band. Northern $C$-band nucleus has multiple components with dominant N-S nucleus and extension at $17^{\circ}$ and $94^{\circ} . \mathrm{NC}=000$.

IR22025+4205 - UGC 11898 has a weak $C$-band elongation at $\mathrm{PA}=-7^{\circ}$. $\mathrm{PA}$ (optical) $=-32^{\circ}$. NC $=000$.

$I R 23135+2517-\mathrm{Zw} 475.056$ has nuclear source within (projected) extended disk of $2.5 \mathrm{kpc}(\mathrm{C} 90 ; \mathrm{C} 91) . \mathrm{NC}=000$.

$I R 23365+3604-$ Point source with $C$-band extension at $\mathrm{PA}=125^{\circ}$. No structure at $L$-band. NC $=000$. 
W. A. Baan and H.-R. Klöckner: Radio properties of FIR-megamaser nuclei, Online Material p 9

Table B.2. Observational classification parameters.

\begin{tabular}{|c|c|c|c|c|c|c|c|c|c|c|c|c|c|c|c|}
\hline Name & & $\begin{array}{c}\text { Redshift } \\
\text { z }\end{array}$ & $\begin{array}{l}\text { Radio } \\
\text { Bands }\end{array}$ & $\begin{array}{c}\text { FIR } \\
\text { Luminosity } \\
L_{\mathrm{FIR}} \\
\left(\log L_{\odot}\right) \\
(4)\end{array}$ & $\begin{array}{c}\text { Radio } \\
\text { Luminosity } \\
L_{\mathrm{r}}(1.4-4.8) \\
\left(\log L_{\odot}\right) \\
(5) \\
\end{array}$ & $\begin{array}{c}\text { Brightness } \\
\text { Temp } \\
T_{\mathrm{b}}(1.4) \\
(\log \mathrm{K}) \\
(6) \\
\end{array}$ & $\begin{array}{c}\text { Brightness } \\
\text { Temp } \\
T_{\mathrm{b}}(4.8) \\
(\log \mathrm{K}) \\
(7) \\
\end{array}$ & $\begin{array}{l}\text { FIR-Radio } \\
\text { Ratio } \\
q(1.4) \\
(\log ) \\
(8) \\
\end{array}$ & $\begin{array}{l}\text { FIR-Radio } \\
\text { Ratio } \\
q(4.8) \\
(\log ) \\
(9) \\
\end{array}$ & $\begin{array}{c}\text { Spectral } \\
\text { Index } \\
\alpha_{\text {total }} \\
\\
\text { (10) } \\
\end{array}$ & $\begin{array}{c}\text { Spectral } \\
\text { Index } \\
\alpha_{\text {peak }} \\
\\
(11) \\
\end{array}$ & $\begin{array}{c}\text { Nuclear } \\
\text { Code } \\
\text { NC }\end{array}$ & $\begin{array}{c}\text { Activity } \\
\text { Factor } \\
\beta_{\mathrm{c}}\end{array}$ & $\begin{array}{c}\text { Optical } \\
\text { Class }\end{array}$ & $\begin{array}{l}\text { Final } \\
\text { Class }\end{array}$ \\
\hline IR00057+4021 & & 0.045 & $\mathrm{LC}$ & 11.32 & $22.48-22.23$ & $4.11 \pm 0.03$ & $4.97 \pm 0.03$ & $2.86 \pm 0.04$ & $3.11 \pm 0.04$ & $0.46 \pm 0.06$ & $.41 \pm 0.06$ & 100 & -0.13 & S2 & A \\
\hline IR01364-1042 & $\mathrm{C}$ & 0.047 & $\mathrm{LC}$ & 11.52 & $22.83-22.72$ & $5.04 \pm 0.02$ & $4.21 \pm 0.00$ & $2.70 \pm 0.03$ & $2.81 \pm 0.03$ & $0.21 \pm 0.03$ & $0.30 \pm 0.030$ & 101 & -0.14 & $\mathrm{C}$ & A \\
\hline IR01418+1651 & & 0.028 & LX & 11.36 & $22.78-22.57$ & $5.01 \pm 0.01$ & $4.04 \pm 0.00$ & $2.59 \pm 0.03$ & $2.81 \pm 0.03$ & $0.40 \pm 0.01$ & $0.56 \pm 0.01$ & 100 & -0.14 & $\mathrm{~L}$ & A \\
\hline IR03260-1422 & & 0.043 & $\mathrm{LC}$ & 11.17 & $22.74-22.35$ & $3.57 \pm 0.02$ & $4.00 \pm 0.02$ & $2.44 \pm 0.03$ & $2.84 \pm 0.03$ & $0.73 \pm 0.04$ & $0.45 \pm 0.05$ & 000 & 0.06 & SB & $\mathrm{S}$ \\
\hline IR04332+0209 & & 0.012 & LC & 10.15 & $20.69-20.63$ & $3.06 \pm 0.08$ & $2.24 \pm 0.06$ & $3.47 \pm 0.07$ & $3.53 \pm 0.05$ & $0.11 \pm 0.15$ & $0.59 \pm 0.27$ & 000 & 1.12 & SB & $S$ \\
\hline IR05414+5840 & $\mathrm{N}$ & 0.016 & $\mathrm{LC}$ & 11.04 & $22.48-21.86$ & $4.33 \pm 0.01$ & $3.35 \pm 0.01$ & $2.56 \pm 0.03$ & $2.98 \pm 0.03$ & $0.71 \pm 0.02$ & $1.02 \pm 0.05$ & 000 & 0.78 & S2 & $S$ \\
\hline IR06206-3646 & & 0.108 & $\mathrm{LC}$ & 11.82 & $23.71-23.52$ & $4.63 \pm 0.02$ & $4.31 \pm 0.02$ & $2.12 \pm 0.03$ & $2.31 \pm 0.03$ & $0.35 \pm 0.04$ & $0.38 \pm 0.04$ & 011 & -0.24 & & A \\
\hline IR08071+0510 & $\mathrm{C}$ & 0.052 & $\mathrm{LC}$ & 11.55 & $23.19-22.67$ & $3.97 \pm 0.01$ & $3.05 \pm 0.01$ & $2.37 \pm 0.03$ & $2.69 \pm 0.03$ & $0.35 \pm 0.02$ & $0.72 \pm 0.04$ & 000 & 0.48 & $\mathrm{C}$ & S \\
\hline IR09320+6134 & & 0.040 & LX & 11.72 & $23.62-23.28$ & $5.14 \pm 0.00$ & $4.65 \pm 0.00$ & $2.10 \pm 0.03$ & $2.45 \pm 0.03$ & $0.64 \pm 0.00$ & $0.71 \pm 0.00$ & 110 & 0.03 & $\mathrm{~L}$ & A \\
\hline IR10173+0828 & & 0.048 & LX & 11.49 & $22.59-22.45$ & $4.34 \pm 0.02$ & $4.25 \pm 0.01$ & $2.91 \pm 0.04$ & $3.05 \pm 0.03$ & $0.26 \pm 0.02$ & $0.29 \pm 0.03$ & 001 & -0.08 & o lines & A \\
\hline IR10378+1109 & & 0.136 & $\mathrm{LC}$ & 12.01 & $23.55-23.25$ & $4.03 \pm 0.11$ & $4.05 \pm 0.03$ & $2.48 \pm 0.10$ & $2.77 \pm 0.04$ & $0.56 \pm 0.18$ & $0.42 \pm 0.23$ & 000 & 0.00 & & $\mathrm{~S}$ \\
\hline IR11010+4107 & & 0.035 & LX & 11.31 & $22.77-22.44$ & $4.05 \pm 0.01$ & $3.77 \pm 0.00$ & $2.55 \pm 0.03$ & $2.88 \pm 0.03$ & $0.62 \pm 0.01$ & $0.61 \pm 0.01$ & 000 & 0.27 & $\mathrm{C}$ & $S$ \\
\hline IR11257+5850 & & 0.010 & LX & 11.38 & $23.10-22.47$ & $5.64 \pm 0.00$ & $4.22 \pm 0.00$ & $2.28 \pm 0.03$ & $2.92 \pm 0.03$ & $1.19 \pm 0.00$ & $0.85 \pm 0.00$ & 100 & 0.40 & $\mathrm{C} / \mathrm{SB}$ & $\mathrm{S}$ \\
\hline IR11506-3851 & & 0.009 & $\mathrm{LC}$ & 10.81 & $22.00-21.70$ & $4.33 \pm 0.02$ & $4.07 \pm 0.01$ & $2.82 \pm 0.03$ & $3.12 \pm 0.03$ & $0.57 \pm 0.03$ & $0.65 \pm 0.04$ & 000 & 0.31 & & S \\
\hline IR12018+1941 & & 0.169 & LC & 12.13 & $23.59-23.26$ & $3.79 \pm 0.04$ & $3.48 \pm 0.04$ & $2.55 \pm 0.05$ & $2.89 \pm 0.04$ & $0.63 \pm 0.09$ & $0.62 \pm 0.10$ & 000 & 0.35 & $\mathrm{~L}$ & $\mathrm{~S}$ \\
\hline \multirow[t]{2}{*}{ IR12112+0305 } & $\mathrm{MN}$ & 0.073 & LX & 12.06 & $23.31-23.04$ & $4.41 \pm 0.01$ & $4.03 \pm 0.00$ & $2.67 \pm 0.03$ & $2.94 \pm 0.03$ & $0.50 \pm 0.01$ & $0.53 \pm 0.01$ & 000 & 0.15 & $\mathrm{C}$ & $\mathrm{S}$ \\
\hline & MS & & LX & & $22.66-22.39$ & $3.84 \pm 0.04$ & $3.02 \pm 0.02$ & $2.67 \pm 0.05$ & $2.94 \pm 0.03$ & $0.51 \pm 0.05$ & $0.66 \pm 0.06$ & 000 & 0.50 & $\mathrm{C}$ & \\
\hline IR12243-0036 & & 0.007 & $\mathrm{LC}$ & 10.63 & $21.56-21.39$ & $5.03 \pm 0.01$ & $5.41 \pm 0.01$ & $3.08 \pm 0.03$ & $3.25 \pm 0.03$ & $0.32 \pm 0.02$ & $0.34 \pm 0.02$ & 101 & -0.25 & S2 & A \\
\hline IR12540+5708 & & 0.042 & LX & 12.11 & $23.92-23.95$ & $5.91 \pm 0.00$ & $5.84 \pm 0.00$ & $2.20 \pm 0.03$ & $2.17 \pm 0.03$ & $-0.05 \pm 0.00$ & $-0.05 \pm 0.00$ & 111 & -1.043 & S1 & A \\
\hline IR13126+2452 & & 0.013 & $\mathrm{LC}$ & 10.84 & $22.01-21.84$ & $5.04 \pm 0.01$ & $4.32 \pm 0.01$ & $2.84 \pm 0.03$ & $3.01 \pm 0.03$ & $0.32 \pm 0.02$ & $0.35 \pm 0.02$ & 101 & -0.05 & SB & A \\
\hline \multirow[t]{2}{*}{ IR13428+5608 } & $\mathrm{N}$ & 0.038 & LX & 11.86 & $23.42-23.13$ & $4.77 \pm 0.00$ & $3.97 \pm 0.00$ & $2.30 \pm 0.03$ & $2.68 \pm 0.03$ & $0.54 \pm 0.00$ & $0.77 \pm 0.00$ & 000 & 0.31 & S2 & $S$ \\
\hline & S & & LX & & $23.04-22.36$ & $3.43 \pm 0.01$ & $3.67 \pm 0.01$ & $2.30 \pm 0.03$ & $2.68 \pm 0.03$ & $1.27 \pm 0.01$ & $0.63 \pm 0.02$ & 000 & 0.25 & S2 & \\
\hline IR14070+0525 & & 0.265 & $\mathrm{LC}$ & 12.50 & $23.98-23.68$ & $3.61 \pm 0.04$ & $2.93 \pm 0.04$ & $2.54 \pm 0.04$ & $2.84 \pm 0.04$ & $0.56 \pm 0.09$ & $0.56 \pm 0.09$ & 000 & 0.41 & & S \\
\hline IR15107+0724 & & 0.013 & $\mathrm{LC}$ & 10.95 & $22.22-22.04$ & $4.93 \pm 0.00$ & $4.39 \pm 0.01$ & $2.74 \pm 0.03$ & $2.93 \pm 0.03$ & $5 \pm 0.02$ & $0.36 \pm 0.02$ & 001 & -0.09 & SB & A \\
\hline IR15250+3609 & & 0.055 & LX & 11.69 & $22.89-22.83$ & $4.77 \pm 0.02$ & $4.63 \pm 0.00$ & $2.81 \pm 0.03$ & $2.88 \pm 0.03$ & $0.13 \pm 0.02$ & $0.14 \pm 0.02$ & 101 & -0.36 & $\mathrm{C}$ & A \\
\hline IR $15327+2340$ & & 0.018 & LX & 11.90 & $23.28-22.85$ & $5.20 \pm 0.00$ & $4.65 \pm 0.00$ & $2.63 \pm 0.03$ & $2.85 \pm 0.03$ & $0.46 \pm 0.00$ & $0.57 \pm 0.00$ & 100 & 0.02 & S2 & S \\
\hline IR16399-0937 & & & $\mathrm{LC}$ & 1.16 & $22.39-22.00$ & $3.20 \pm 0.03$ & $3.50 \pm 0.03$ & $2.78 \pm 0.03$ & $3.17 \pm 0.03$ & $1 \pm 0.05$ & $.47 \pm 0.07$ & 000 & 0.29 & $\mathrm{~L} / \mathrm{SB}$ & $\mathrm{S}$ \\
\hline IR17208-0014 & & & $\mathrm{LC}$ & 11.38 & $22.78-22.53$ & $4.72 \pm 0.02$ & & & & & & & 0.16 & $\mathrm{~L}$ & $\mathrm{~S}$ \\
\hline IR20550+1656 & & 0.037 & $\mathrm{LC}$ & 11.60 & $22.64-22.25$ & $3.16 \pm 0.04$ & $2.60 \pm 0.04$ & $2.97 \pm 0.04$ & $3.36 \pm 0.05$ & $0.73 \pm 0.08$ & $0.71 \pm 0.17$ & 000 & 0.78 & SB & $\mathrm{S}$ \\
\hline IR22025+4205 & & 0.016 & $\mathrm{LC}$ & 10.80 & $22.05-21.80$ & $4.46 \pm 0.04$ & $3.48 \pm 0.01$ & $2.77 \pm 0.04$ & $3.01 \pm 0.03$ & $0.45 \pm 0.06$ & $0.88 \pm 0.07$ & 000 & 0.63 & $\mathrm{C}$ & S \\
\hline IR22088-1831 & & 0.170 & $\mathrm{LC}$ & 12.11 & $23.78-23.80$ & $3.06 \pm 0.04$ & $4.08 \pm 0.02$ & $2.34 \pm 0.04$ & $2.32 \pm 0.03$ & $-0.04 \pm 0.06$ & $-0.27 \pm 0.08$ & 011 & -0.78 & & A \\
\hline IR22491-1808 & & 0.076 & LX & 11.86 & $22.84-22.62$ & $3.24 \pm 0.03$ & $3.96 \pm 0.01$ & $3.03 \pm 0.04$ & $3.25 \pm 0.03$ & $0.41 \pm 0.04$ & $0.28 \pm 0.05$ & 001 & 0.04 & & S \\
\hline IR23135+2517 & & 0.027 & LX & 11.23 & $22.54-22.04$ & $3.74 \pm 0.01$ & $3.91 \pm 0.01$ & $2.70 \pm 0.03$ & $3.20 \pm 0.03$ & $0.94 \pm 0.01$ & $0.69 \pm 0.02$ & 000 & 0.41 & S2 & $\mathrm{S}$ \\
\hline IR23365+3604 & & 0.064 & $\mathrm{LC}$ & 11.86 & $23.29-22.93$ & $4.69 \pm 0.02$ & $3.86 \pm 0.01$ & $2.58 \pm 0.03$ & $2.95 \pm 0.03$ & $0.68 \pm 0.03$ & $0.80 \pm 0.04$ & 000 & 0.45 & $\mathrm{C}$ & $\mathrm{S}$ \\
\hline \multicolumn{16}{|l|}{ Unconfirmed } \\
\hline IR00335-2732 & & 0.069 & LC & 11.66 & $23.16-22.84$ & $4.36 \pm 0.03$ & $3.65 \pm 0.02$ & $2.51 \pm 0.04$ & $2.83 \pm 0.04$ & $0.59 \pm 0.05$ & $0.66 \pm 0.06$ & 000 & 0.33 & $\mathrm{C}$ & S \\
\hline IR01314+0941 & & 0.049 & LC & 11.12 & $22.68-22.19$ & $3.80 \pm 0.02$ & $3.31 \pm 0.03$ & $2.45 \pm 0.03$ & $2.93 \pm 0.04$ & $0.91 \pm 0.05$ & $0.85 \pm 0.07$ & 000 & 0.62 & & S \\
\hline IR02483+4302 & & 0.052 & $\mathrm{LC}$ & 11.49 & $24.71-24.81$ & $6.82 \pm 0.01$ & $6.84 \pm 0.01$ & $0.78 \pm 0.03$ & $0.69 \pm 0.03$ & $-0.18 \pm 0.02$ & $-0.17 \pm 0.02$ & 111 & -1.84 & $\mathrm{~L}$ & A \\
\hline IR03056+2034 & & 0.027 & $\mathrm{LC}$ & 10.91 & $22.14-21.57$ & $4.22 \pm 0.02$ & $3.20 \pm 0.04$ & $2.78 \pm 0.03$ & $3.35 \pm 0.04$ & $1.05 \pm 0.07$ & $1.00 \pm 0.07$ & 000 & 0.91 & SB & $S$ \\
\hline IR03119+1448 & & 0.076 & $\mathrm{LC}$ & 11.49 & $22.58-22.32$ & $3.28 \pm 0.09$ & $3.10 \pm 0.06$ & $2.93 \pm 0.08$ & $3.19 \pm 0.05$ & $0.49 \pm 0.16$ & $0.55 \pm 0.18$ & 000 & 0.46 & & S \\
\hline IR10485-1447 & & 0.133 & $\mathrm{LC}$ & 11.88 & 23.39-23.11 & $4.11 \pm 0.04$ & $3.80 \pm 0.04$ & $2.50 \pm 0.05$ & $2.78 \pm 0.04$ & $0.53 \pm 0.09$ & $0.49 \pm 0.10$ & 000 & 0.13 & & S \\
\hline IR11069+2711 & $\mathrm{C}$ & 0.070 & LC & 11.48 & $23.21-22.59$ & $3.67 \pm 0.02$ & $2.54 \pm 0.03$ & $2.29 \pm 0.03$ & $2.56 \pm 0.03$ & $0.33 \pm 0.05$ & $1.02 \pm 0.11$ & 000 & 0.84 & SB & S \\
\hline \multirow[t]{2}{*}{ IR13097-1531 } & NW & 0.022 & $\mathrm{LC}$ & 11.19 & $22.12-21.57$ & $3.16 \pm 0.03$ & $2.86 \pm 0.03$ & $2.56 \pm 0.034$ & $3.06 \pm 0.04$ & $1.01 \pm 0.07$ & $0.83 \pm 0.09$ & 000 & 0.74 & SB & S \\
\hline & $\mathrm{C}$ & & $\mathrm{LC}$ & & $22.48-21.99$ & $3.15 \pm 0.02$ & $2.18 \pm 0.01$ & $2.56 \pm 0.03$ & $3.06 \pm 0.03$ & $0.90 \pm 0.03$ & $1.27 \pm 0.08$ & 000 & 1.31 & SB & \\
\hline IR13254+4754 & & 0.059 & $\mathrm{LC}$ & 11.24 & $22.77-22.33$ & $3.89 \pm 0.03$ & $3.58 \pm 0.04$ & $2.48 \pm 0.04$ & $2.92 \pm 0.04$ & $0.82 \pm 0.07$ & $0.79 \pm 0.08$ & 000 & 0.50 & $\mathrm{C}$ & $\mathrm{S}$ \\
\hline IR13451+1232 & & 0.121 & $\mathrm{LC}$ & 11.86 & $26.21-25.97$ & $7.54 \pm 0.01$ & $7.17 \pm 0.01$ & $-0.34 \pm 0.03$ & $-0.10 \pm 0.03$ & $0.46 \pm 0.03$ & $0.48 \pm 0.03$ & 110 & -1.55 & S1.5 & A \\
\hline IR15233+0533 & & 0.055 & $\mathrm{LC}$ & 11.41 & $22.92-22.50$ & $3.68 \pm 0.04$ & $3.54 \pm 0.03$ & $2.50 \pm 0.04$ & $2.92 \pm 0.04$ & $0.78 \pm 0.07$ & $0.75 \pm 0.08$ & 000 & 0.47 & $\mathrm{~L}$ & $\mathrm{~S}$ \\
\hline \multirow[t]{3}{*}{ IR $17526+3253$} & $\mathrm{~N}$ & 0.025 & $\mathrm{LC}$ & 10.80 & $22.72-21.89$ & $2.68 \pm 0.02$ & $2.90 \pm 0.02$ & $1.90 \pm 0.03$ & $2.61 \pm 0.03$ & $1.55 \pm 0.05$ & $0.25 \pm 0.22$ & 001 & 0.59 & SB & $\mathrm{S}$ \\
\hline & SE2 & & $\mathrm{LC}$ & & $22.39-21.69$ & $3.61 \pm 0.05$ & $1.46 \pm 0.03$ & $1.90 \pm 0.05$ & $2.61 \pm 0.04$ & $1.30 \pm 0.09$ & $1.52 \pm 0.44$ & 000 & 1.55 & SB & \\
\hline & SE3 & & $\mathrm{LC}$ & & $21.47-21.50$ & $1.13 \pm 0.30$ & $1.26 \pm 0.05$ & $2.90 \pm 0.33$ & $2.61 \pm 0.05$ & $-0.06 \pm 0.62$ & $1.29 \pm 0.80$ & 000 & 1.40 & SB & \\
\hline IR20491+1846 & & 0.029 & $\mathrm{LC}$ & 10.86 & $21.87-21.07$ & $2.58 \pm 0.11$ & $1.40 \pm 0.19$ & $3.00 \pm 0.10$ & $3.80 \pm 0.19$ & $1.50 \pm 0.38$ & $1.49 \pm 0.91$ & 000 & 1.91 & SB & $\mathrm{S}$ \\
\hline IR23050+0359 & & 0.047 & $\mathrm{LC}$ & 11.35 & $23.26-22.43$ & $3.09 \pm 0.01$ & $3.70 \pm 0.02$ & $2.10 \pm 0.03$ & $2.92 \pm 0.03$ & $1.54 \pm 0.03$ & $0.41 \pm 0.06$ & 000 & 0.11 & SB & $S$ \\
\hline
\end{tabular}


Table B.2. continued.

\begin{tabular}{|c|c|c|c|c|c|c|c|c|c|c|c|c|c|c|}
\hline Name & $\begin{array}{c}\text { Redshift } \\
\text { z }\end{array}$ & $\begin{array}{l}\text { Radio } \\
\text { Bands }\end{array}$ & $\begin{array}{c}\text { FIR } \\
\text { Luminosity } \\
L_{\mathrm{FIR}} \\
\left(\log L_{\odot}\right) \\
(4)\end{array}$ & $\begin{array}{c}\text { Radio } \\
\text { Luminosity } \\
L_{\mathrm{r}}(1.4-4.8) \\
\left(\log L_{\odot}\right) \\
(5)\end{array}$ & $\begin{array}{c}\text { Brightness } \\
\text { Temp } \\
T_{\mathrm{b}}(1.4) \\
(\log \mathrm{K}) \\
(6)\end{array}$ & $\begin{array}{c}\text { Brightness } \\
\text { Temp } \\
T_{\mathrm{b}}(4.8) \\
(\log \mathrm{K}) \\
\text { (7) }\end{array}$ & $\begin{array}{l}\text { FIR-Radio } \\
\text { Ratio } \\
q(1.4) \\
(\log ) \\
(8)\end{array}$ & $\begin{array}{c}\text { FIR-Radio } \\
\text { Ratio } \\
q(4.8) \\
(\log ) \\
(9)\end{array}$ & $\begin{array}{c}\text { Spectral } \\
\text { Index } \\
\alpha_{\text {total }} \\
\\
(10)\end{array}$ & $\begin{array}{c}\text { Spectral } \\
\text { Index } \\
\alpha_{\text {peak }} \\
\\
(11)\end{array}$ & $\begin{array}{c}\text { Nuclear } \\
\text { Code } \\
\text { NC } \\
\\
(12)\end{array}$ & $\begin{array}{c}\text { Activity } \\
\text { Factor } \\
\beta_{\mathrm{c}} \\
\\
(13)\end{array}$ & $\begin{array}{c}\text { Optical } \\
\text { Class }\end{array}$ & $\begin{array}{l}\text { Final } \\
\text { Class }\end{array}$ \\
\hline \multicolumn{15}{|l|}{ OH Absorbers } \\
\hline IR14566-1629 & 0.012 & $\mathrm{LC}$ & 10.36 & $23.46-23.15$ & $6.83 \pm 0.01$ & $5.79 \pm 0.01$ & $0.90 \pm 0.03$ & $1.22 \pm 0.03$ & $0.59 \pm 0.02$ & $0.65 \pm 0.03$ & 110 & -0.67 & S2 & A \\
\hline IR $16577+5900$ & 0.019 & LX & 11.00 & $22.66-21.94$ & $3.79 \pm 0.01$ & $3.36 \pm 0.00$ & $2.35 \pm 0.03$ & $3.07 \pm 0.03$ & $1.35 \pm 0.01$ & $0.93 \pm 0.02$ & 000 & 0.72 & $\mathrm{C}$ & S \\
\hline
\end{tabular}

Column (1) - Galaxy or IRAS name. The designations M, N, S, E, W, and C identify the Main, North, South, East, West, and Central components.

Column (2) - Redshift. The linear scale at the distance of the source is given by $19.39 \times z \mathrm{kpc}$ per arcsecond.

Column (3) - Spectral code of two observing frequencies where $L$ stands for $L$-band (1.43 GHz), C for $C$-band (4.85 GHz), and $S$ for $S$-band $(8.44 \mathrm{GHz})$.

Column (4) - The integrated FIR luminosity $L(\mathrm{FIR})=4 \pi D_{L}^{2} \mathrm{~S}_{\mathrm{FIR}}$, where $D_{L}$ is the cosmological distance and where $\mathrm{S}_{\mathrm{FIR}}$ is the integrated FIR flux in the band from $40 \mu \mathrm{m}$ and $120 \mu \mathrm{m}$ such that $\mathrm{S}_{\mathrm{FIR}}=1.26 \times 10^{-14}\left(2.58 S_{60 \mu \mathrm{m}}+S_{100 \mu \mathrm{m}}\right) \mathrm{W} \mathrm{m}^{-2}$. The FIR flux densities are in units of Jy.

Column (5) - The integrated $1.4 \mathrm{GH}$ and $4.85 \mathrm{GHz}$ spectral luminosity $L$ (radio) $=4 \pi D_{L}^{2} S_{1.4 / 4.8 \mathrm{GHz}} \mathrm{W} \mathrm{Hz}{ }^{-1}$, where $D_{L}$ is the cosmological distance and where $S_{1.4 / 4.8 \mathrm{GHz}}$ is the flux density at 1.4 and $4.85 \mathrm{GHz}$. The observing bandwidth for the VLA-A observations was $50 \mathrm{MHz}$. For sources with data at $8.44 \mathrm{GHz}$ (from CHYT) taken at $0.25^{\prime \prime}$ resolution, the $4.85 \mathrm{GHz}$ flux density has been found by interpolation between 1.43 and $8.44 \mathrm{GHz}$.

Columns (6 and 7) - Logarithm of the Rayleigh-Jeans brightness temperature $T_{\mathrm{b}}$ using the integrated fluxes at $1.43 \mathrm{GHz}$ and at $4.85 \mathrm{or} 8.44 \mathrm{GHz}$ defined as: $T_{\mathrm{b}}=\left(c^{2} S_{\text {radio }} / 2 \mathrm{k} v^{2}\right)\left(8 \ln 2 / 3 \pi \theta_{M} \theta_{m}\right)$. A uniform elliptical source component has been assumed with FWHM fitted angular diameters $\theta_{M}, \theta_{m}$ and integrated flux density $S_{\text {radio }}$ as given in Cols. 6-8 of Table 1.

Columns (8 and 9) - The FIR-radio luminosity ratio at 1.43 and $4.85 \mathrm{GHz}$. The $q$-ratio is defined as $q=\log \left(3.36 \times 10^{2}\left(2.58 S_{60} \mu \mathrm{m}+\right.\right.$ $\left.S_{100 \mu \mathrm{m}}\right) / S_{\text {radio }}$ ) with the FIR fluxes in Jy and the integrated radio flux density in mJy (Helou et al. 1985; CHYT). The $q$-ratio for sources with $8.44 \mathrm{GHz}$ data has been interpolated to represent the radio flux at $4.85 \mathrm{GHz}$. The $q$-values for identifiable radio components have been based on the unresolved FIR fluxes and the sum of the radio fluxes of the components.

Columns (10 and 11) - The integrated and peak nuclear spectral index defined as $\alpha=-\mathrm{d}(\ln S) / \mathrm{d}(\ln v)$ using the integrated and peak flux densities of Table 1 (Cols. 7 and 6). This spectral index is taken between $1.43 \mathrm{GHz}$ and $4.85 \mathrm{GHz}$ or $8.44 \mathrm{GHz}$. The spectral index based on the peak flux densities is given for completeness. These peak spectral indices will be overestimates when the nuclear sources are resolved at higher frequencies. Identifiable structural components have been designated as separate entries.

Column (12) - Nuclear classification code for the dominant power source for the nuclear emission. This "NC" code consists of three digits with a " 1 " indicating a tendency towards AGN activity and a "0" towards SBN activity. The three digits of the radio nuclear activity code are respectively based on the criteria of brightness temperature at $4.85 \mathrm{GHz}$, the FIR-radio ratio at $4.85 \mathrm{GHz}$, and the integrated spectral index (see Sect. 3 for further discussion).

Column (13) Weighted nuclear Activity Factor $\beta_{\mathrm{c}}$ based on the three radio diagnostic parameters. Positive values indicate a SBN signature for the dominant nuclear power source and negative values indicate an AGN signature.

Column (14) The optical classification of the nuclear emission taken from Baan et al. (1998). The following classifications are used: S1, S2 and S1.5 = Seyfert types 1,2 , and $1.5, \mathrm{SB}=$ Starburst Nucleus, $\mathrm{L}=$ LINER, and C = Composite spectrum.

Column (15) A final classification for the dominant power source for the nuclear activity as $\mathrm{A}(\mathrm{GN})$ and $\mathrm{S}(\mathrm{BN})$. 